\title{
Unveiling the Effects of Foreign Exchange Intervention: A Panel Approach
}




\section{WP/15/130}

\section{IMF Working Paper}

\section{Unveiling the Effects of Foreign Exchange Intervention: A Panel Approach}

by Gustavo Adler, Noemie Lisack and Rui C. Mano

IMF Working Papers describe research in progress by the author(s) and are published to elicit comments and to encourage debate. The views expressed in IMF Working Papers are those of the author(s) and do not necessarily represent the views of the IMF, its Executive Board, or IMF management.
I $N$
E P
NATIONAL
$M O N E T A R Y$
F U N D 


\title{
IMF Working Paper
}

\author{
Research Department
}

\section{Unveiling the Effects of Foreign Exchange Intervention: A Panel Approach* Prepared by Gustavo Adler ${ }^{\dagger}$, Noemie Lisack ${ }^{\ddagger}$ and Rui C. Mano ${ }^{\dagger}$}

Authorized for distribution by Steve Phillips

June 2015

IMF Working Papers describe research in progress by the author(s) and are published to elicit comments and to encourage debate. The views expressed in IMF Working Papers are those of the author(s) and do not necessarily represent the views of the IMF, its Executive Board, or IMF management.

\begin{abstract}
We study the effect of foreign exchange intervention on the exchange rate relying on an instrumental-variables panel approach. We find robust evidence that intervention affects the level of the exchange rate in an economically meaningful way. A purchase of foreign currency of 1 percentage point of GDP causes a depreciation of the nominal and real exchange rates in the ranges of [1.7-2.0] percent and [1.4-1.7] percent respectively. The effects are found to be quite persistent. The paper also explores possible asymmetric effects, and whether effectiveness depends on the depth of domestic financial markets.
\end{abstract}

JEL Classification Numbers: E58, F31

Keywords: foreign exchange intervention, exchange rate, reserves

Authors’ E-Mail Addresses: gadler@imf.org; rmano@imf.org

\footnotetext{
$\bar{\dagger}$ International Monetary Fund; ${ }^{*}$ European University Institute. The views expressed in this paper are those of the authors and do not necessarily represent those of their institutions or their institutions' policies.

* We are grateful to Steven Phillips, Tam Bayoumi, Mitali Das, Jaebin Ahn, Ahuja Ashvin, Emine Boz, Varapat Chensavasdijai, Mai Dao, Martin Kaufman, Ruy Lama, Nan Li, Kenji Moriyama, Helene Poirson, Belen Sbrancia, Viktor Tsyrennikov, Mauricio Vargas and participants in the EUI Macroeconomics Working Group for their feedback. Remaining errors are ours.
} 


\section{Introduction}

Growing international financial integration and volatile global financial conditions have posed significant challenges to policy makers across the world in recent years. Emerging market countries, and increasingly advanced market economies, have resorted to a battery of policy tools, including macro-prudential measures, capital controls and foreign exchange intervention (FXI) to cope with the effects of large capital flows. However, the merits of these different tools remain under debate. There is some consensus on how these instruments should impact key macroeconomic variables in theory, but the empirical evidence on their effect and economic relevance remains elusive. The evidence has been particularly hard to find in the case of FXI, reflecting serious endogeneity issues that hamper the identification of its effects, especially on the exchange rate. This is the focus of our paper.

Large changes in central banks' net foreign asset positions over the last two decades, primarily driven by FXI, give testimony of the importance of FXI as a macroeconomic management tool (Figure 1). Whether countries deployed FXI as a way to accumulate reserves for precautionary reasons (e.g., Aizenman and Lee, 2008; Jeanne and Rancière, 2011; Ghosh et al., 2012) or seeking to manage their exchange rates (e.g. Reinhart and Reinhart, 2008; Aizenman and Lee, 2008; Adler and Tovar, 2014; Gagnon, 2012a) was a much discussed subject during the 2000s. ${ }^{1}$ But, with the implementation of unconventional monetary policies in advanced economies in recent years, countries facing large capital inflows have been more vocal and open about the primary objectives of carrying out FXI operations, namely dampening the effects of these inflows on their exchange rates. Indeed, a simple indicator of the degree of exchange rate management (Figure 2) points to a wide range of de facto exchange rate regimes (even among de jure floaters), suggesting that many countries have relied heavily on sterilized FXI to manage their exchange rates. ${ }^{2}$ However, the effectiveness of sterilized FXI in terms of its impact on the level of the exchange rate remains debatable. ${ }^{3}$

From a theoretical perspective, the literature has proposed two channels through which FXI can affect exchange rates: a signaling and a portfolio balance channel. On one hand, sterilized FXI can affect the exchange rate by providing information about the central bank's monetary policy intentions (signaling). On the other hand, the portfolio balance theory, pioneered by Henderson and Rogoff (1982), Kouri (1983) and Branson and Henderson (1985), and further studied recently by Kumhof (2010) and Gabaix and Maggiori (2014), established that FXI can affect the exchange rate when domestic and external assets are imperfect substitutes. In this case, sterilized intervention increases the relative supply of domestic assets, driving risk premia up and thereby exerting depreciation pressures on the exchange rate. ${ }^{4,5}$

\footnotetext{
${ }^{1}$ For studies on the motives of FXI, see also Canales-Kriljenko (2003), Moreno (2005), Neely (2008), Stone et al. (2009).

${ }^{2}$ The indicator of exchange rate management is defined as: $\rho_{j} \equiv \sigma_{j}^{N F A} /\left(\sigma_{j}^{N F A}+\sigma_{j}^{S}\right)$ where $\sigma_{j}^{N F A}$ and $\sigma_{j}^{S}$ denote the standard deviations of a proxy of FXI and of the nominal exchange rate, respectively. $\rho_{j}$ varies between 0 and 1 , corresponding to a pure floating and a peg respectively.

${ }^{3} \mathrm{~A}$ number of studies have explored the effects of FXI on exchange rate volatility and obtained more conclusive results than those focused on exchange rate levels. See for example, Stone et al. (2009), Mandeng (2003), Kamil (2008), Pattanaik and Sahoo (2003), Domaç and Mendoza (2004), Guimarães Filho and Karacadağ (2004), Abenoja (2003). Another related strand of the literature has studied the impact of FXI (and more generally net official flows) on the current account. See for example, Bayoumi et al. (2014), Gagnon (2012b) and Gagnon (2013).

${ }^{4}$ In the case of the signaling channel, FXI serves as tool to convey information about policy intentions, and thus cannot be thought of as an independent policy instrument. In the case of imperfect asset substitutability (portfolio
} 
Figure 1: Central Bank Net Foreign Asset Position, 1996-

2013

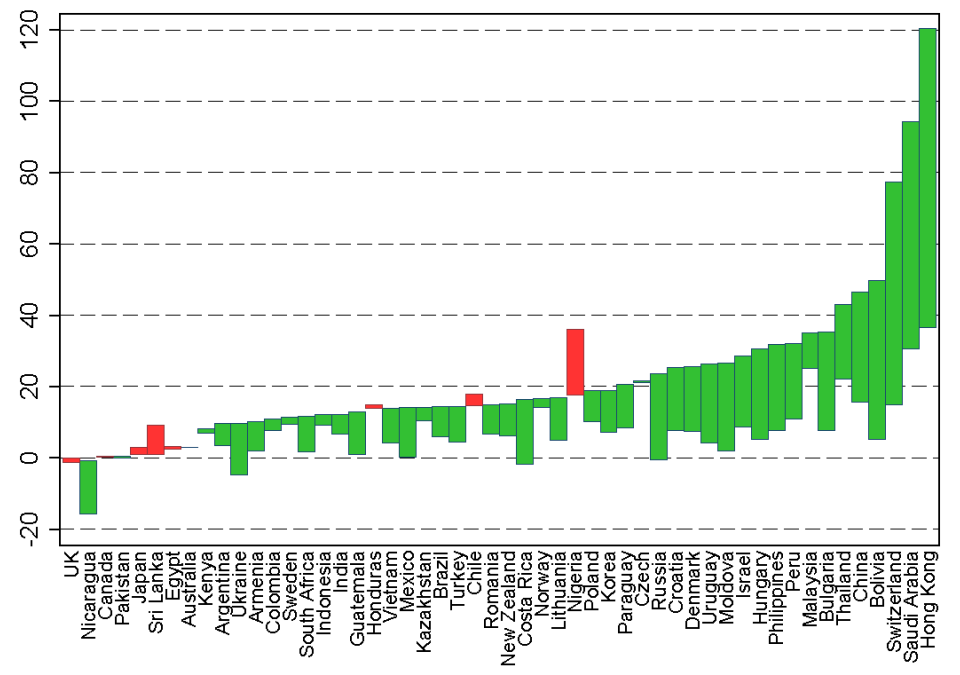

Sources: IMF International Financial Statistics; and authors' calculations. Green (red) bars indicate an increase (drop) in the NFA position during the period. The lower end of the bar indicates the initial position, and the upper end the final position, for the cases of NFA increases; and the opposite for NFA decreases.

From an empirical point of view, the literature has seen two distinct waves of work. During the 1980s and part of the 1990s, numerous studies focused on cases of FXI in advanced economies (see, for example, the extensive work by Dominguez, 1990, 1998, Dominquez and Kenen, 1992, Dominguez and Frankel, 1990, 1993b, 1993a; Ghosh, 1992; Kaminsky and Lewis, 1996; and Kenen, 1982). ${ }^{6}$ In general, these studies found limited evidence of effectiveness with regard to the level of the exchange rate, unless interventions were coordinated across major central banks. More recently, in an effort to study developments in emerging markets, there has been a number of studies with more supportive, although often mixed, evidence that intervention affects the exchange rate (see recent reviews of the literature in Menkhoff, 2010 and 2013). Most of these studies, however, are country-specific and thus their results are difficult to generalize. In fact, robust evidence on the effects of FXI has been hard to find beyond some specific cases, possibly reflecting the endogeneity of FXI decisions, which tends to conceal the effect of FXI on exchange rates. ${ }^{7}$ Even when authors have been able to overcome endogeneity issues, the employed empirical strategies have often fallen short of shedding light on the macroeconomic relevance of such effects. Specifically, most of the attempts

balance channel), however, FXI is an additional, independent, policy tool.

${ }^{5}$ The literature often also refers to a microstructure channel, according to which, frictions at a micro level can affect the extent to which information embedded in central bank operations (assuming an informational advantage) reaches market participants and shapes their expectations. See, for example, Lyons (2006).

${ }^{6}$ For comprehensive reviews of the early literature, focused on advanced economies, see Sarno and Taylor (2001) and Neely (2005).

${ }^{7}$ This is illustrated in Appendix Figure A1, which displays the bivariate relationship between a proxy of FXI and changes in the nominal exchange on a monthly basis for the period 1996-2013 and a large set of countries. As shown, if anything, there is a positive relationship indicating that positive FXI (reserve accumulation) is accompanied by exchange rate appreciation. 
Figure 2: Degree of Exchange Rate Management, 1996-2013

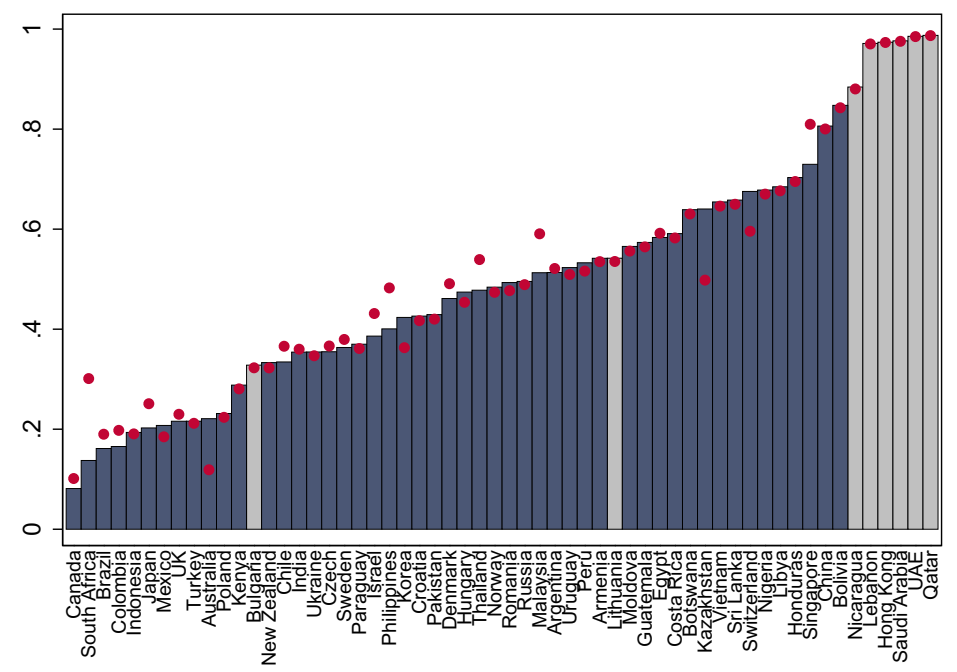

Sources: IMF International Financial Statistics; and authors' calculations. Bars report a measure $\rho_{j} \equiv \sigma_{j}^{N F A} /\left(\sigma_{j}^{N F A}+\sigma_{j}^{S}\right)$ where $\sigma_{j}^{N F A}$ and $\sigma_{j}^{S}$ denote the standard deviations of changes in net foreign assets and of the nominal exchange rate, respectively. Gray bars correspond to countries with de-jure pegs for most of the sample period (1996-2013), and blue bars otherwise. Dots report to a similar measure of exchange rate management but based on a proxy of FXI that includes non-spot interventions (see Annex 1).

have relied on high-frequency data (including intra-day) in order to mitigate reverse causality (see, for example, Tapia and Tokman, 2004; Guimarães Filho and Karacadağ, 2004; Domaç and Mendoza, 2004; Humala and Rodriguez, 2010; Kamil, 2008; Rincón and Toro, 2011; Dominguez et al., 2013; etc.). This approach (sometimes combined with some form of instrumentation) helped to break the contemporaneous relationship between exchange rate movements and FXI decisions, by exploiting the fact that FXI decisions are normally taken at a lower frequency than exchange rate movements. In general, this 'high-frequency' approach has been successful in finding evidence of effects of FXI on the exchange rate in the short-run, but doubts remain as to whether these effects are sufficiently persistent (i.e., beyond a few days) to have relevant macroeconomic implications. Another shortcoming of these papers has been their focus on one country at a time, preventing a generalization of the results and exploring time-invariant country characteristics. Other papers have relied on lower frequency data, taking an instrumental-variables approach to overcome endogeneity. This approach has had mixed results, arguably reflecting different degrees of success in finding good instruments. Examples of this approach are the work in Phillips et al. (2013) — who explore determinants of exchange rates in a panel setting, although without focusing on FXI —Daude et al. (2014) - who explore the effects of FXI on exchange rates in a panel setting, although relying on an unconventional measure of FX intervention; and Blanchard et al. (2015) — who study the effect of FXI in the context of capital flow shocks.

Our approach entails exploring the effect of FXI on exchange rate levels in an instrumentalvariables panel setting, relying on a large set of countries (52) and a long time period (monthly data for 1996-2013). The large sample allows us to draw more general conclusions from our findings, and the relatively low frequency of the data allows to study the relevance of the effects of FXI from 
a macroeconomic point of view.

We find robust evidence that intervention affects the level of the exchange rate in a macroeconomic meaningful way. A positive FXI (FX purchase) of 1 percentage point of GDP leads to, depending on the specification, a depreciation of the nominal and real exchange rate in the range of [1.7-2.0] percent and [1.4-1.5] respectively. Furthermore, we find these effects to be persistent, with estimates of their half-life in the range of [12-23] months, again depending on the specification. The paper explores possible asymmetric effects, and finds no indication of different effects between positive and negative intervention, suggesting that FXI is a useful policy tool both when facing appreciation and depreciation pressures.

The rest of the paper is organized as follows: Section 2 presents the econometric methodology, the main results and a number of robustness checks. Section 3 discusses extensions of the benchmark specification, exploring dynamic and asymmetric effects. Section 4 concludes with the key takeaways.

\section{Econometric Analysis}

\subsection{Approach}

The main challenge in evaluating the impact of FXI on exchange rates is the endogeneity of interventions to exchange rate movements. To overcome this difficulty, we use a two-stage least squares approach with instrumental variables that relies on identifying exogenous variations in FXI (i.e. interventions that are unrelated to contemporaneous exchange rate movements). Specifically, we employ the following specification, which is estimated in a panel setting:

$$
\begin{aligned}
\log \left(E R_{i t}\right) & =\alpha+\beta \log \left(E R_{i t-1}\right)+\gamma \widehat{F X I} I_{i t}+\boldsymbol{\delta}^{\prime} \mathbf{X}_{i t}+\eta_{i}+\epsilon_{i t} \\
F X I_{i t} & =a+b \log \left(E R_{i t-1}\right)+\mathbf{c}^{\prime} \mathbf{Z}_{i t}+\mathbf{d}^{\prime} \mathbf{X}_{i t}+u_{i}+v_{i t}
\end{aligned}
$$

Equation (1) is the second-stage exchange rate equation linking the exchange rate $\left(E R_{i t}\right)$ to exogenous (instrumented) FXI as well as to a series of regressors $\left(X_{i t}\right)$ that are expected to drive exchange rate variations. Equation (2) is the first-stage regression relating FXI to a series of driving factors, including those introduced in the second stage and a full set of instruments $\left(Z_{i t}\right)$. $E R_{i t}$ denotes country $i$ 's exchange rate at time $t$ (either nominal or real bilateral, in both cases vis-a-vis the U.S. dollar, or real effective, depending on the specification). We follow the convention that an increase represents an appreciation of the domestic currency in all cases; ${ }^{8} F X I_{i t}$ stands for our proxy of foreign exchange intervention; $Z_{i t}$ is the set of instrumental variables; and $X_{i t}$ is a set of control variables; all of which are discussed in detail next. $u_{i}$ and $\eta_{i}$ denote country fixed effects for the first and second stage regressions, respectively.

Our interest lies primarily on the parameter $\gamma$. A value of $\gamma<0$ would indicate that a positive intervention (buying foreign currency) depreciates the domestic currency. The benchmark specification assumes homogeneous parameters across countries, although later we relax this assumption to assess the robustness of the results. Next, we discuss how the measure of FXI, and the sets instruments and controls are constructed.

\footnotetext{
${ }^{8}$ In the rest of the paper, results refer to real bilateral exchange rates vis-a-vis the U.S., unless otherwise indicated. This minimizes issues related to episodes of high inflation (that would affect nominal rate more markedly); and allows for a more parsimonious mapping between the regressors and the dependent variable.
} 


\section{Foreign Exchange Intervention}

The definition of FXI is not straightforward, although this is rarely discussed in the literature. From the perspective of the portfolio balance channel, FXI should be understood as any policyinduced financial operation that changes the foreign exchange position of the public sector, as changes in the net FX position would imply changes in the relative supply of domestic assets (money if unsterilized, central bank notes or T-bills if sterilized).$^{9}$ In practice, however, measuring FXI is a difficult task, on account of several issues.

First, ideally one would measure FXI as any policy-induced changes in the FX position of the consolidated public sector. ${ }^{10}$ However, such data is rarely available, particularly at high frequency. We therefore focus on the central bank's balance sheet, in line with the literature. ${ }^{11}$ Second, data on actual purchases and sales of foreign assets are not generally reported. Thus, we conduct our study using several different proxies for FXI. In the baseline estimations, we simply rely on the change in the (net) foreign asset position of the central bank as a proxy for FXI. Later we conduct a number of robustness checks, with other proxies, including correcting for valuation effects and off-balance sheet operations (i.e. swaps, forward, etc.). We normalize FXI by (HP filtered) trend GDP in U.S. dollars, in order to prevent endogeneity arising from movements in the U.S. dollar value of nominal GDP. This normalization facilitates the interpretation and comparability of results across countries and time; and helps to prevent unit root problems. In section 2.3 we also discuss alternative normalizations. Finally, as we are interested in the effect of sterilized interventions, we control for the monetary policy stance by including the domestic (and foreign) interest rate in all specifications.

\section{Exchange Rate Determinants (Controls)}

Guided by the vast literature on exchange rate determinants, we include in the specification a series of controls, which we classify into two groups: a small set of controls, aimed at keeping the specification as parsimonious as possible; and an expanded set of standard exchange rate determinants, the inclusion of which tends to restrict the sample due to data availability.

The small set of control variables is composed of (i) the level of the Chicago Board Options Exchange Market Volatility Index (VIX); (ii) three indices of commodity prices (for energy, metals and agriculture products) with country-specific coefficients; and (iii) the interest rate differential visa-vis the U.S., as we focus on the bilateral exchange rate with the US Dollar in most specifications. Commodity prices are included to capture possible terms of trade shocks at high frequency and their differentiated impacts depending on whether countries export or import different commodities. Introducing the interest rate in the model is key for interpreting the results as pertaining to sterilized intervention, since it allows us to control for the effect of simultaneous changes in the interest rate on the exchange rate (which could come from non-sterilized intervention). This approach is superior to trying to exclude observations of unsterilized interventions, since it does not require to define arbitrary thresholds for classifying FXI as sterilized or non-sterilized. Various robustness checks are

\footnotetext{
${ }^{9} \mathrm{~A}$ definition from the perspective of the signaling channel is less straightforward, as one could think of a broader set of policies that would provide information about monetary policy intentions.

${ }^{10}$ Whether public sector agencies other than the central bank intend to affect the exchange rate or not with their FX transactions is irrelevant for the analysis of the effects of FXI on the exchange rate, since the portfolio balance channel would operate in either case. Intent, however, may be relevant for assessing the appropriateness of policies, but such analysis is beyond the scope of this paper.

${ }^{11}$ In most cases, central bank FX transactions appear to be the major source of public sector FX transactions, except in some countries with sizeable sovereign wealth funds.
} 
conducted later, including by introducing the domestic and foreign interest rates separately. When using the nominal bilateral exchange rate, the inflation differential vis-a-vis the US is added in order to control for movements in the exchange rate that reflect persistently high inflation levels. ${ }^{12}$

The expanded set of controls includes other exchange rate determinants following Phillips et al. (2013). These are slow moving series or variables for which less data is available: GDP per capita and expected GDP growth (both relative to the U.S.), lagged trade balance, and trade openness. Finally, we also include in the expanded set of controls an additional variable that captures global financial conditions (in addition to the interest rate and the VIX index) as measured by the net portfolio flows to other countries in the sample, reported by Emerging Portfolio Fund Research (EPFR).

\section{Instruments}

Key to our methodology is finding suitable instrumental variables, i.e. variables that are strongly correlated with FXI, but not with the exchange rate. We explore a number of potential instruments related to various motives for conducting FXI, primarily related to reserve accumulation for precautionary reasons and exchange rate stabilization motives related to balance sheet effects.

For the first case (FXI for precautionary motives), we examine usual reserve coverage metrics, as in other studies. ${ }^{13}$ These include lagged gross (net) international reserves relative to GDP, imports, external debt or M2 - that aim to capture exogenous precautionary motives. All variables are evaluated in levels as well as relative to the average of other countries in the sample, the latter intending to unveil possible 'keeping up with the Joneses' effects. ${ }^{14}$

We also explore a set of new instrumental variables related to exchange rate stabilization motives, not used in previous studies. In particular, we focus on the interaction between a measure of the degree of deposit dollarization in the domestic financial system, and different measures of exogenous financial shocks (VIX, Global EMBI, EPFR flows, etc.) that would tend to exert depreciation pressures on most EMEs. A negative statistical link between this variable and FXI would indicate the use of intervention in response to exogenous shocks with the aim of mitigating balance sheet effects.

Finally, we also examine lagged trade balance as a possible instrument related to a mercantilist motive.

Most of these variables gave intuitive results, with different degrees of significance. However, the instruments finally included in the baseline estimations were chosen according to three specific criteria:

i. Each individual instrumental variable delivers a coefficient sign in the first stage regression consistent with economic theory. This is aimed at ensuring that the variable actually reflects the intervention motive in mind.

\footnotetext{
${ }^{12}$ By excluding each country at a time, we ensure the measure is exogenous from the perspective of each small open economy. See Blanchard et al. (2015) for a similar approach.

${ }^{13}$ See, for example, Phillips et al. (2013) and Daude et al. (2014).

${ }^{14}$ The variables are also included in levels — which significance would indicate an objective to build up buffers - and in first differences — which would indicate a desire to maintain a certain level of reserves in proportion to such variables.
} 
ii. The variable helps mitigate endogeneity bias in the second stage regression. That is, the coefficient for fitted values of FXI in the second stage regression should be lower than in the simple OLS version. This condition is imposed because, as discussed before, the direction of the bias is known (central banks tend to react in order to dampen movements in the exchange rate, rather than the opposite, which implies, if any, an upward bias in an OLS regression-where exchange rates are defined as US\$/LC). ${ }^{15}$

iii. When instrumenting jointly (using all variables that satisfy the two previous criteria), the specification is required to pass the overidentification and weak instrument tests. The first one (also known as Hansen or J-test) examines the null hypothesis of joint validity of the instruments. ${ }^{16}$ To test for weak instruments, we follow the test developed by Stock and Yogo $(2002) \cdot{ }^{17}$

\section{Data}

The dataset encompasses monthly observations for 52 countries (13 advanced and 39 emerging market economies), during the period January 1996-October 2013 (see Annex Table A1 for more details). The sample focuses on countries with their own legal tender (i.e., excludes countries that use other countries' currencies as legal tender). Periods during which the de-facto exchange rate regimes (following the classification of Ilzetzki et al., 2010) are classified as freely falling or dual exchange rates are excluded. Data come from the IMF's International Financial Statistics, World Economic Outlook and Direction of Trade Statistics. Interest rates are obtained from Datastream, expected GDP growth are from Consensus Forecast. The different measures of the size of the domestic financial sector (discussed below) come from the World Bank's Global Financial Development Database (GFDD). EPFR flows are obtained from Haver Analytics. Deposits dollarization is obtained from the database constructed by Yeyati (2006) and complemented by recent data from IMF country desks. Capital control indices come from Schindler (2009), Chinn and Ito (2006) and Quinn and Toyoda (2008), and are extended in some cases using the IMF's Annual Report on Exchange Arrangements and Exchange Restrictions (AREAER) data. Details of variables' construction and sources are summarized in Appendix Table A2.

In the benchmark specification we exclude the $1 \%$ top and bottom observations ${ }^{18}$ for each variable (except for variables bounded by definition) to avoid undue influence of possible outliers in the results. Table 1 presents summary statistics of the main variables; and the distribution of FXI is shown in Appendix Figure A2. ${ }^{19}$

\footnotetext{
${ }^{15}$ This is different from studies that focus on the effect of FXI on the current account, where the direction of the endogeneity bias is ambiguous. See, for example, Bayoumi et al. (2014).

${ }^{16}$ Its statistic is built on the weighted sum of the residuals from the exclusion restrictions and follows a $\chi^{2}$ in the number of overidentifying restrictions. For instruments to pass this test, the test statistic should be low enough not to reject the null hypothesis.

${ }^{17}$ The test is built on the $\mathrm{F}$ statistic of the first stage regression, but specifically tailored to weak instruments issues, and is therefore preferable to the often used rule of thumb of the first stage $\mathrm{F}$ statistic being larger than 10 . Instruments are considered weak when the maximum size of the IV coefficient's bias relative to the OLS coefficient's bias exceeds a certain threshold. The null hypothesis assumes that instruments are weak, and critical values which depend on the number of instruments used are tabulated in Stock and Yogo (2002). Given the number of instruments included in our benchmark specification, a test statistic above 18 (11) rejects a relative bias above 5\% (10\%).

${ }^{18}$ The $1^{\text {st }}$ and $99^{\text {th }}$ percentiles are taken over the cross-country distribution for the whole sample. For the exchange rates, we excluded outliers based on the distribution of their month-to-month growth rate.

${ }^{19}$ Variables are tested for stationarity. Both nominal and real bilateral exchange rates are stationary; while real effective exchange rates display non-stationarity in some cases, and under some tests. See further discussion in the robustness section.
} 
Table 1: Descriptive statistics

\begin{tabular}{|c|c|c|c|c|c|}
\hline & Obs & Mean & Std Dev & Min & Max \\
\hline $\log$ RER & 9149 & -3.14 & 2.57 & -10.16 & 0.69 \\
\hline $\log$ NER & 9149 & -3.03 & 2.47 & -9.96 & 0.73 \\
\hline $\log$ REER & 8597 & 4.53 & 0.15 & 3.81 & 5.08 \\
\hline FXI/GDP & 9149 & 0.19 & 0.71 & -2.70 & 3.77 \\
\hline FXI/GDP (valuation adj) & 9012 & 0.12 & 0.69 & -2.74 & 3.45 \\
\hline FXI/GDP (valuation adj + off-BS) & 9011 & 0.12 & 0.71 & -3.04 & 3.84 \\
\hline FXI/GDP ( $\Delta$ Reserves $)$ & 9103 & 0.18 & 0.66 & -2.40 & 3.50 \\
\hline FXI/M2 (instrumented) & 9053 & 0.53 & 2.20 & -9.09 & 13.21 \\
\hline FXI/Financial sector size (IFS, instrumented) & 8741 & 0.39 & 1.44 & -5.57 & 9.67 \\
\hline FXI/Financial sector size (WB, instrumented) & 8424 & 0.45 & 1.85 & -9.15 & 14.13 \\
\hline FXI/Market size (instrumented) & 8515 & 2.27 & 14.17 & -65.66 & 152.99 \\
\hline VIX & 9149 & 21.53 & 7.75 & 10.42 & 59.89 \\
\hline Interest rate (differential) & 9149 & 0.39 & 0.51 & -0.39 & 5.12 \\
\hline Inflation rate (differential) & 8723 & 3.09 & 4.99 & -4.71 & 49.75 \\
\hline Change in M2/GDP & 9149 & 0.49 & 0.83 & -1.90 & 4.47 \\
\hline Financial dollarization & 9149 & 23.45 & 23.24 & 0.00 & 92.60 \\
\hline Import coverage & 9141 & 0.52 & 0.49 & -0.44 & 4.31 \\
\hline Low import coverage & 9149 & 0.22 & 0.42 & 0.00 & 1.00 \\
\hline Broad money coverage & 9146 & 0.39 & 0.40 & -1.42 & 2.16 \\
\hline GDP per capita (differential) & 9046 & -1.32 & 0.83 & -3.03 & 0.27 \\
\hline Expected GDP growth (differential) & 8571 & 1.08 & 2.54 & -14.36 & 11.02 \\
\hline Trade Balance & 9039 & -0.29 & 1.01 & -3.70 & 3.57 \\
\hline Trade Openness & 9046 & 5.43 & 3.04 & 1.41 & 27.68 \\
\hline EPFR/GDP & 9149 & 0.01 & 0.11 & -0.59 & 0.46 \\
\hline EMBI spread & 8421 & 4.78 & 2.54 & 1.51 & 14.19 \\
\hline
\end{tabular}

\subsection{Main results}

Table 2 presents the second stage OLS and IV results for the baseline specification, using only the set of instrumental variables that pass the criteria discussed above. In each case, results are reported for the nominal bilateral, real bilateral, and real effective exchange rates, both using the small and the expanded set of controls. Table 3 displays the results of the first stage regression for the same specifications (for the IV specifications alone).

As discussed previously, OLS regressions (Table 2, columns 1-6) deliver counter-intuitive results, due to the endogeneity of FXI, even after controlling for usual exchange rate drivers. Our instrumentation strategy (columns 7-12), on the other hand, delivers coefficients for FXI in the second stage that are highly significant and with the expected sign. Moreover, the magnitude of the effects is economically meaningful. A FXI of 1 percentage point of GDP leads to a depreciation in the range of [1.7-1.9] percent of the nominal exchange rate, depending on the specification. The effect is somewhat smaller on the real bilateral exchange rate, in the range of [1.4-1.5] percent, possibly indicating some exchange rate pass-through to inflation; and more so on the real effective exchange rate ([1.2-1.3] percent), suggesting that simultaneous FXI in trading partners tend to offset each other. The results on the real effective exchange rate, however, should be interpreted with caution as regressors are not measured in relative terms to trading partners. Thus, we focus on the first two exchange rate measures, for which the mapping with the regressors is clear. Control variables in the second stage all have expected signs and are statistically significant, except for the interest rate differential. We conduct a series of robustness checks on the latter in the section 2.3. The set of variables found to be valid instruments include: 
i. Change in M2 normalized by trend nominal GDP, consistent with findings in a number of recent papers (e.g., Phillips et al., 2013; Daude et al., 2014) and the precautionary motives highlighted by Obstfeld et al. (2010).

ii. Broad money coverage: NFA/M2 (lagged)

iii. Imports coverage measured by NFA/imports (lagged), where we also find a non-linear relationship, with a shift for low import coverage levels.

iv. Financial dollarization interacted with VIX.

\subsection{Main Robustness Checks}

We conduct a number of robustness checks on our baseline specification. In this section, we pay special attention to (i) the normalization of FXI (and the link between FXI and domestic financial deepening); (ii) the instrumentation; (iii) the exchange rate regime; (iv) other simultaneous policy responses; and (v) the proxy of FXI.

\section{Different normalizations}

While normalizing FXI by GDP seems intuitive and helps interpret the magnitudes involved, there is no obvious economic case for using this particular measure. In fact, the theory of FXI points to other potentially better normalizations. From the perspective of the portfolio balance channel, a relevant measure would be the amount of intervention relative to, for example, the supply of domestic assets or the size of domestic financial markets. ${ }^{20,21}$ We explore four alternative norms that go in such direction:

i. broad money (M2);

ii. a (narrow) measure of the size of the domestic financial sector, based on the total amount of domestic assets held by domestic banks (excluding the central bank) as reported in IFS. ${ }^{22}$

iii. an alternative (broader) measure of the size of the domestic financial system based on data from the World Bank Global Financial Development Database (GFDD). This measure encompasses domestic assets of domestic banks, non-bank financial institutions, pension funds, mutual funds, and insurance companies.

iv. a measure of the size of the domestic financial market, also based on GFDD data, encompassing stock market capitalization, and the value of outstanding domestic private and public debt securities. ${ }^{23}$

\footnotetext{
${ }^{20}$ The desirable normalization from the perspective of the signaling channel is less obvious, since the effect on the exchange rate could be driven by the mere FXI announcement by the central bank; with actual amounts arguably playing a secondary role.

${ }^{21}$ Other studies have explored measures of FXI relative to the foreign exchange market turnover. However, this is difficult to implement in a panel setting due to limited data on the latter.

${ }^{22}$ Non-depositary financial corporations are also excluded due to missing information for many countries.

${ }^{23}$ The latter dataset contains many missing values for some countries. Long-term moving averages are computed to mitigate the limited data.
} 
Table 2: Second stage, baseline results

\begin{tabular}{|c|c|c|c|c|c|c|c|c|c|c|c|c|}
\hline \multicolumn{13}{|c|}{ Dependent variable: exchange rate } \\
\hline & $(1)$ & $(2)$ & (3) & $(4)$ & $(5)$ & $\overline{(6)}$ & $(7)$ & $(8)$ & (9) & $(10)$ & $(11)$ & $(12)$ \\
\hline Exchange rate definition: & Nominal & Real & Real effective & Nominal & Real & Real effective & Nominal & Real & Real effective & Nominal & Real & Real effective \\
\hline FXI/GDP & $\begin{array}{c}0.751^{* * *} \\
(0.118)\end{array}$ & $\begin{array}{c}0.740^{* * *} \\
(0.123)\end{array}$ & $\begin{array}{l}0.105^{* *} \\
(0.048)\end{array}$ & $\begin{array}{c}0.805^{* * *} \\
(0.120)\end{array}$ & $\begin{array}{c}0.781^{* * * *} \\
(0.122)\end{array}$ & $\begin{array}{c}0.143^{* * *} \\
(0.049)\end{array}$ & & & & & & \\
\hline FXI/GDP (instrumented) & & & & & & & $\begin{array}{c}-1.692^{* * *} \\
(0.358)\end{array}$ & $\begin{array}{c}-1.430^{* * *} \\
(0.326)\end{array}$ & $\begin{array}{c}-1.218^{* * *} \\
\quad(0.250)\end{array}$ & $\begin{array}{c}-1.905^{* * *} \\
(0.460)\end{array}$ & $\begin{array}{c}-1.544^{* * *} \\
(0.390)\end{array}$ & $\begin{array}{c}-1.343^{* * *} \\
(0.331)\end{array}$ \\
\hline Dependent variable (lagged) & $\begin{array}{c}0.980^{* * *} \\
(0.005)\end{array}$ & $\begin{array}{c}0.975^{* * *} \\
(0.004)\end{array}$ & $\begin{array}{c}0.969^{* * *} \\
(0.004)\end{array}$ & $\begin{array}{c}0.976^{* * *} \\
(0.006)\end{array}$ & $\begin{array}{c}0.960^{* * *} \\
(0.006)\end{array}$ & $\begin{array}{c}0.965^{* * *} \\
(0.005)\end{array}$ & $\begin{array}{c}0.976^{* * * *} \\
(0.003)\end{array}$ & $\begin{array}{c}0.966^{* * * *} \\
(0.004)\end{array}$ & $\begin{array}{c}0.961^{* * *} \\
(0.004)\end{array}$ & $\begin{array}{c}0.975^{* * *} \\
(0.003)\end{array}$ & $\begin{array}{c}0.953^{* * * *} \\
(0.004)\end{array}$ & $\begin{array}{c}0.958^{* * * *} \\
(0.005)\end{array}$ \\
\hline VIX & $\begin{array}{c}-0.037^{* * *} \\
(0.006)\end{array}$ & $\begin{array}{c}-0.025^{* * *} \\
(0.006)\end{array}$ & $\begin{array}{l}-0.004 \\
(0.005)\end{array}$ & $\begin{array}{c}-0.040^{* * *} \\
(0.006)\end{array}$ & $\begin{array}{c}-0.031^{* * *} \\
(0.006)\end{array}$ & $\begin{array}{l}-0.007 \\
(0.006)\end{array}$ & $\begin{array}{c}-0.058^{* * *} \\
(0.006)\end{array}$ & $\begin{array}{c}-0.043^{* * *} \\
(0.005)\end{array}$ & $\begin{array}{c}-0.014^{* * *} \\
(0.005)\end{array}$ & $\begin{array}{c}-0.065^{* * *} \\
(0.007)\end{array}$ & $\begin{array}{c}-0.050^{* * *} \\
(0.006)\end{array}$ & $\begin{array}{c}-0.020^{* * *} \\
(0.006)\end{array}$ \\
\hline Interest rate (differential) & $\begin{array}{c}0.004 \\
(0.138)\end{array}$ & $\begin{array}{c}0.212 \\
(0.138)\end{array}$ & $\begin{array}{l}0.233^{*} \\
(0.118)\end{array}$ & $\begin{array}{c}0.064 \\
(0.148)\end{array}$ & $\begin{array}{c}0.341^{* *} \\
(0.147)\end{array}$ & $\begin{array}{l}0.339^{* *} \\
(0.140)\end{array}$ & $\begin{array}{c}-0.324^{* *} \\
(0.137)\end{array}$ & $\begin{array}{c}0.014 \\
(0.117)\end{array}$ & $\begin{array}{c}0.088 \\
(0.117)\end{array}$ & $\begin{array}{c}-0.340^{* *} \\
(0.161)\end{array}$ & $\begin{array}{c}0.105 \\
(0.134)\end{array}$ & $\begin{array}{c}0.177 \\
(0.134)\end{array}$ \\
\hline Inflation rate (differential) & $\begin{array}{l}0.027^{* *} \\
(0.011)\end{array}$ & & & $\begin{array}{l}0.028^{* *} \\
(0.013)\end{array}$ & & & $\begin{array}{c}0.050^{* * * *} \\
(0.012)\end{array}$ & & & $\begin{array}{c}0.056^{* * *} \\
(0.014)\end{array}$ & & \\
\hline GDP per capita (differential) & & & & $\begin{array}{c}3.121^{* * * *} \\
(0.830)\end{array}$ & $\begin{array}{c}4.869^{* * *} \\
(1.070)\end{array}$ & $\begin{array}{l}1.490^{*} \\
(0.745)\end{array}$ & & & & $\begin{array}{c}0.911 \\
(1.012)\end{array}$ & $\begin{array}{c}3.533^{* * *} \\
(0.957)\end{array}$ & $\begin{array}{c}0.400 \\
(0.722)\end{array}$ \\
\hline Expected GDP growth (differential) & & & & $\begin{array}{l}0.048^{* *} \\
(0.021)\end{array}$ & $\begin{array}{l}0.053^{* *} \\
(0.021)\end{array}$ & $\begin{array}{c}0.057^{* * *} \\
(0.015)\end{array}$ & & & & $\begin{array}{c}0.120^{* * *} \\
(0.029)\end{array}$ & $\begin{array}{c}0.113^{* * *} \\
(0.026)\end{array}$ & $\begin{array}{c}0.095^{* * *} \\
(0.021)\end{array}$ \\
\hline Trade Balance (lagged) & & & & $\begin{array}{c}-0.108^{*} \\
(0.056)\end{array}$ & $\begin{array}{c}-0.255^{* * *} \\
(0.070)\end{array}$ & $\begin{array}{c}0.047 \\
(0.059)\end{array}$ & & & & $\begin{array}{l}0.175^{*} \\
(0.105)\end{array}$ & $\begin{array}{l}-0.032 \\
(0.091)\end{array}$ & $\begin{array}{c}0.245^{* * *} \\
(0.082)\end{array}$ \\
\hline Trade Openness & & & & $\begin{array}{c}0.068 \\
(0.057)\end{array}$ & $\begin{array}{l}0.136^{*} \\
(0.076)\end{array}$ & $\begin{array}{l}-0.056 \\
(0.041)\end{array}$ & & & & $\begin{array}{c}0.276^{* * *} \\
(0.101)\end{array}$ & $\begin{array}{c}0.303^{* * *} \\
(0.090)\end{array}$ & $\begin{array}{c}0.069 \\
(0.068)\end{array}$ \\
\hline EPFR/GDP & & & & $\begin{array}{c}1.447^{* * *} \\
(0.289)\end{array}$ & $\begin{array}{c}1.680^{* * *} \\
(0.327)\end{array}$ & $\begin{array}{c}0.746^{* *} \\
(0.312)\end{array}$ & & & & $\begin{array}{c}1.613^{* * *} \\
(0.318)\end{array}$ & $\begin{array}{c}1.859^{* * *} \\
(0.312)\end{array}$ & $\begin{array}{c}0.911^{* * *} \\
(0.239)\end{array}$ \\
\hline Commodity prices & Yes & Yes & Yes & Yes & Yes & Yes & Yes & Yes & Yes & Yes & Yes & Yes \\
\hline Country fixed effects & Yes & Yes & Yes & Yes & Yes & Yes & Yes & Yes & Yes & Yes & Yes & Yes \\
\hline $\begin{array}{l}\text { Observations } \\
\text { Countries }\end{array}$ & $\begin{array}{c}8755 \\
55\end{array}$ & $\begin{array}{c}9149 \\
52\end{array}$ & $\begin{array}{c}9017 \\
55\end{array}$ & $\begin{array}{c}7950 \\
51\end{array}$ & $\begin{array}{c}8312 \\
51\end{array}$ & $\begin{array}{c}7871 \\
51\end{array}$ & $\begin{array}{c}8755 \\
55\end{array}$ & $\begin{array}{c}9149 \\
52\end{array}$ & $\begin{array}{c}9017 \\
55\end{array}$ & $\begin{array}{c}7950 \\
51\end{array}$ & $\begin{array}{c}8312 \\
51\end{array}$ & $\begin{array}{c}7871 \\
51\end{array}$ \\
\hline $\begin{array}{l}\text { Countries } \\
R^{2}\end{array}$ & $\begin{array}{c}55 \\
0.99\end{array}$ & $\begin{array}{c}52 \\
0.99\end{array}$ & $\begin{array}{c}55 \\
0.98\end{array}$ & $\begin{array}{c}51 \\
0.99\end{array}$ & $\begin{array}{c}51 \\
0.98\end{array}$ & $\begin{array}{c}51 \\
0.98\end{array}$ & $\begin{array}{c}55 \\
0.98\end{array}$ & $\begin{array}{c}52 \\
0.98\end{array}$ & $\begin{array}{c}55 \\
0.97\end{array}$ & $\begin{array}{c}51 \\
0.98\end{array}$ & $\begin{array}{c}51 \\
0.98\end{array}$ & $\begin{array}{c}51 \\
0.97\end{array}$ \\
\hline $\mathrm{J}$ p-value & & & & & & & 0.30 & 0.39 & 0.38 & 0.39 & 0.44 & 0.05 \\
\hline Stock \& Yogo stat & & & & & & & 17.99 & 19.42 & 17.13 & 12.82 & 14.77 & 12.19 \\
\hline
\end{tabular}

Standard errors robust to autocorrelation and heteroskedasticity in parentheses

${ }^{*} p<0.10,{ }^{* *} p<0.05,{ }^{* * *} p<0.01$ 
Table 3: First stage, baseline results

Dependent variable: foreign exchange intervention (\% GDP)

\begin{tabular}{|c|c|c|c|c|c|c|}
\hline Exchange rate definition: & $\begin{array}{c}(1) \\
\text { Nominal }\end{array}$ & $\begin{array}{c}(2) \\
\text { Real }\end{array}$ & $\begin{array}{c}(3) \\
\text { Real effective }\end{array}$ & $\begin{array}{c}(4) \\
\text { Nominal }\end{array}$ & $\begin{array}{c}(5) \\
\text { Real }\end{array}$ & $\begin{array}{c}(6) \\
\text { Real effective }\end{array}$ \\
\hline Exchange rate (lagged) & $\begin{array}{l}-0.001 \\
(0.001)\end{array}$ & $\begin{array}{c}-0.003^{* *} \\
(0.001)\end{array}$ & $\begin{array}{c}-0.005^{* * *} \\
(0.002)\end{array}$ & $\begin{array}{c}0.000 \\
(0.001)\end{array}$ & $\begin{array}{c}-0.002 \\
(0.001)\end{array}$ & $\begin{array}{c}-0.004^{* *} \\
(0.002)\end{array}$ \\
\hline VIX & $\begin{array}{c}-0.006^{* *} \\
(0.002)\end{array}$ & $\begin{array}{c}-0.005^{* *} \\
(0.002)\end{array}$ & $\begin{array}{c}-0.004^{*} \\
(0.002)\end{array}$ & $\begin{array}{c}-0.006^{* *} \\
(0.002)\end{array}$ & $\begin{array}{c}-0.005^{* *} \\
(0.002)\end{array}$ & $\begin{array}{c}-0.004^{*} \\
(0.002)\end{array}$ \\
\hline Interest rate (differential) & $\begin{array}{c}-0.130^{* * *} \\
(0.042)\end{array}$ & $\begin{array}{c}-0.095^{* *} \\
(0.037)\end{array}$ & $\begin{array}{c}-0.116^{* *} \\
(0.046)\end{array}$ & $\begin{array}{c}-0.148^{* * *} \\
(0.039)\end{array}$ & $\begin{array}{c}-0.108^{* * *} \\
(0.036)\end{array}$ & $\begin{array}{c}-0.113^{* *} \\
(0.043)\end{array}$ \\
\hline Inflation rate (differential) & $\begin{array}{c}0.007^{* *} \\
(0.003)\end{array}$ & & & $\begin{array}{c}0.008^{* *} \\
(0.003)\end{array}$ & & \\
\hline Change in M2/GDP & $\begin{array}{c}0.103^{* * *} \\
(0.023)\end{array}$ & $\begin{array}{c}0.104^{* * *} \\
(0.022)\end{array}$ & $\begin{array}{c}0.103^{* * *} \\
(0.025)\end{array}$ & $\begin{array}{c}0.085^{* * *} \\
(0.019)\end{array}$ & $\begin{array}{c}0.089^{* * *} \\
(0.019)\end{array}$ & $\begin{array}{c}0.076^{* * *} \\
(0.019)\end{array}$ \\
\hline Financial dollarization $\times$ VIX & $\begin{array}{l}-0.009^{*} \\
(0.005)\end{array}$ & $\begin{array}{c}-0.011^{* *} \\
(0.005)\end{array}$ & $\begin{array}{l}-0.010 \\
(0.007)\end{array}$ & $\begin{array}{l}-0.010^{*} \\
(0.006)\end{array}$ & $\begin{array}{l}-0.011^{*} \\
(0.005)\end{array}$ & $\begin{array}{c}-0.016^{* *} \\
(0.007)\end{array}$ \\
\hline Import coverage (lagged) & $\begin{array}{c}-0.282^{* *} \\
(0.129)\end{array}$ & $\begin{array}{l}-0.252^{*} \\
(0.142)\end{array}$ & $\begin{array}{l}-0.222 \\
(0.134)\end{array}$ & $\begin{array}{c}-0.271^{*} \\
(0.141)\end{array}$ & $\begin{array}{l}-0.275^{*} \\
(0.149)\end{array}$ & $\begin{array}{l}-0.236 \\
(0.147)\end{array}$ \\
\hline Low import coverage (lagged) & $\begin{array}{c}0.049 \\
(0.044)\end{array}$ & $\begin{array}{c}0.035 \\
(0.042)\end{array}$ & $\begin{array}{c}0.041 \\
(0.042)\end{array}$ & $\begin{array}{c}0.003 \\
(0.040)\end{array}$ & $\begin{array}{l}-0.010 \\
(0.038)\end{array}$ & $\begin{array}{c}0.020 \\
(0.040)\end{array}$ \\
\hline Broad money coverage (lagged) & $\begin{array}{c}0.018 \\
(0.140)\end{array}$ & $\begin{array}{l}-0.021 \\
(0.145)\end{array}$ & $\begin{array}{l}-0.098 \\
(0.150)\end{array}$ & $\begin{array}{l}-0.051 \\
(0.158)\end{array}$ & $\begin{array}{l}-0.060 \\
(0.157)\end{array}$ & $\begin{array}{l}-0.131 \\
(0.151)\end{array}$ \\
\hline GDP per capita (differential) & & & & $\begin{array}{c}-0.672^{* *} \\
(0.289)\end{array}$ & $\begin{array}{l}-0.482 \\
(0.333)\end{array}$ & $\begin{array}{c}-0.698^{*} \\
(0.367)\end{array}$ \\
\hline Expected GDP growth (differential) & & & & $\begin{array}{c}0.025^{* * *} \\
(0.008)\end{array}$ & $\begin{array}{c}0.024^{* * *} \\
(0.007)\end{array}$ & $\begin{array}{c}0.026^{* * *} \\
(0.008)\end{array}$ \\
\hline Trade Balance (lagged) & & & & $\begin{array}{c}0.109^{* * *} \\
(0.039)\end{array}$ & $\begin{array}{l}0.100^{* *} \\
(0.038)\end{array}$ & $\begin{array}{c}0.134^{* * *} \\
(0.043)\end{array}$ \\
\hline Trade Openness & & & & $\begin{array}{c}0.076^{* *} \\
(0.038)\end{array}$ & $\begin{array}{l}0.071^{*} \\
(0.037)\end{array}$ & $\begin{array}{c}0.090^{* *} \\
(0.039)\end{array}$ \\
\hline EPFR/GDP & & & & $\begin{array}{c}0.094 \\
(0.073)\end{array}$ & $\begin{array}{c}0.107 \\
(0.072)\end{array}$ & $\begin{array}{l}0.140^{*} \\
(0.070)\end{array}$ \\
\hline Commodity prices & Yes & Yes & Yes & Yes & Yes & Yes \\
\hline Country fixed effects & Yes & Yes & Yes & Yes & Yes & Yes \\
\hline Observations & 8755 & 9149 & 9017 & 7950 & 8312 & 7871 \\
\hline Countries & 55 & 52 & 55 & 51 & 51 & 51 \\
\hline$R^{2}$ & 0.11 & 0.10 & 0.10 & 0.12 & 0.11 & 0.10 \\
\hline F stat & 7.32 & 8.18 & 6.94 & 6.25 & 7.14 & 6.13 \\
\hline F p-value & 0.00 & 0.00 & 0.00 & 0.00 & 0.00 & 0.00 \\
\hline
\end{tabular}


In general, the correlation across different measures is high, except for the relationship between FXI/M2 and the other metrics (see Appendix Table A3). As shown in Table 4, results with these alternative metrics have the expected sign and are statistically significant in all cases. First stage results are present in Appendix Table A4. The magnitude of the coefficient on FXI cannot be directly compared across columns, because of the different scale across FXI measures. However, the marginal effect with respect to FXI/GDP, when using norm J, can be derived as:

$$
\frac{\partial \log \left(E R_{i t}\right)}{\partial F X I_{i t}^{G D P}}=\gamma \frac{G D P_{i t}}{\text { Norm }_{i t}}
$$

Evaluated at the mean value of $G D P_{i t} / N o r m J_{i t}$, the marginal effect of $F X I^{G D P}$ is very similar to the baseline estimation (see last row of Table 4). Furthermore, equation (3) would suggest that, as expected, the effect of $F X I^{G D P}$ on the exchange rate is smaller the larger is the domestic financial market (any of the alternative metrics).

Table 4: Different normalizations, second stage

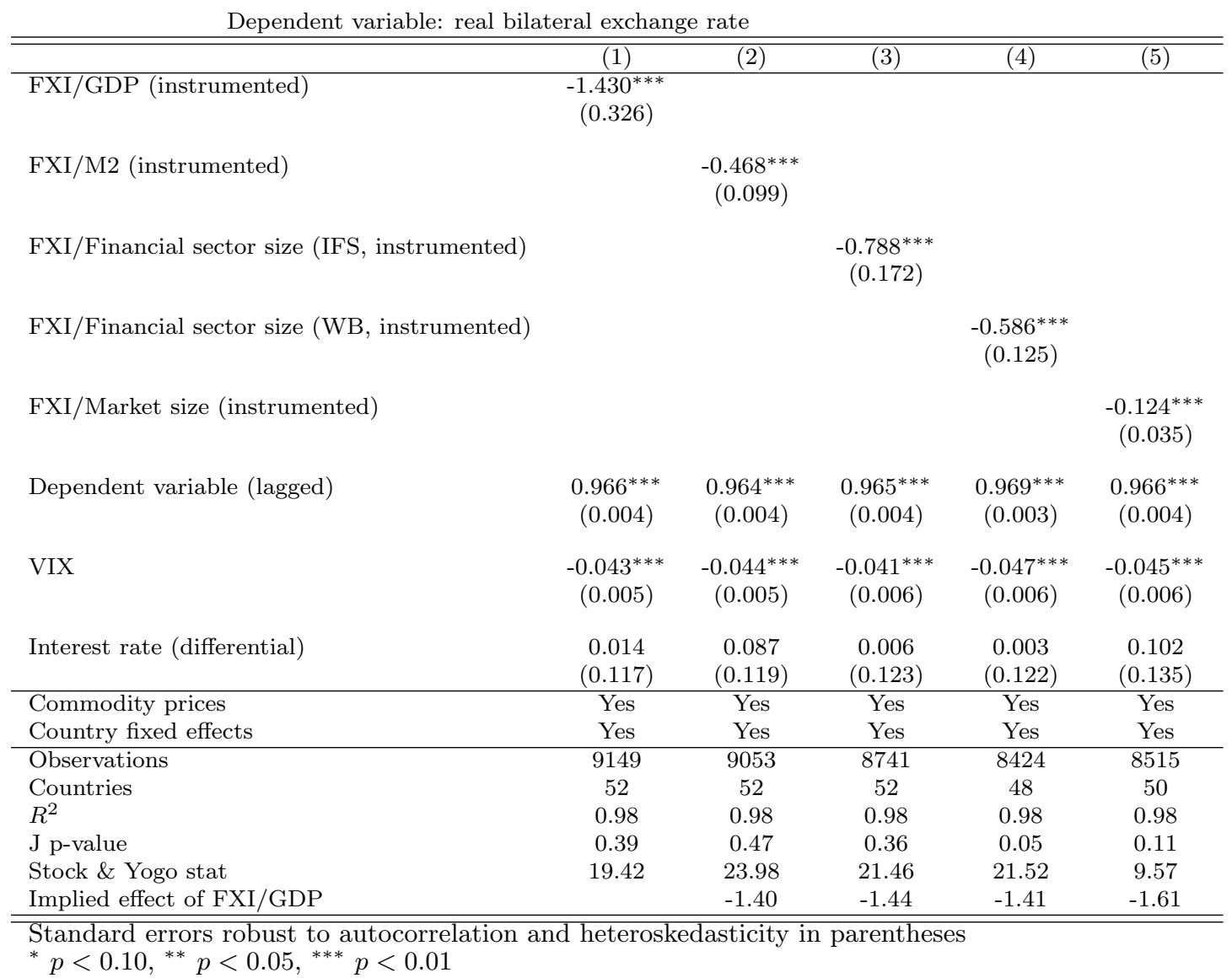

To formally test the presence of decreasing effects, however, two changes are necessary in the baseline specification: (i) introducing $F X I^{G D P}$ and $F X I^{\text {NormJ }}$ simultaneously in the second stage regression; and (ii) instrumenting both measures since the particular measure of exogenous FXI might depend on the normalization used. ${ }^{24}$ Thus, we run:

\footnotetext{
${ }^{24}$ We use the same instruments regardless of normalization, allowing first stage coefficients (on the instruments and controls) to vary across normalizations. This approach follows from treating each normalization of FXI as a different endogenous variable.
} 


$$
\begin{aligned}
\log \left(E R_{i t}\right) & =\alpha+\beta \log \left(E R_{i t-1}\right)+\gamma_{1} \widehat{F X I_{i t}^{G D}}+\gamma_{2} F \widehat{F I_{i t}^{N o r m} J}+\boldsymbol{\delta}^{\prime} \mathbf{X}_{i t}+\eta_{i}+\epsilon_{i t} \\
F X I_{i t}^{G D P} & =a_{1}+b_{1} \log \left(E R_{i t-1}\right)+\mathbf{c}_{1}^{\prime} \mathbf{Z}_{i t}+\mathbf{d}_{1}^{\prime} \mathbf{X}_{i t}+u_{i}+v_{i t} \\
F X I_{i t}^{N o r m J} & =a_{2}+b_{2} \log \left(E R_{i t-1}\right)+\mathbf{c}_{2}^{\prime} \mathbf{Z}_{i t}+\mathbf{d}_{2}^{\prime} \mathbf{X}_{i t}+w_{i}+z_{i t}
\end{aligned}
$$

In this setting, the marginal effect of $F X I_{i t}^{G D P}$ on the exchange rate is given by:

$$
\frac{\partial \log \left(E R_{i t}\right)}{\partial F X I_{i t}^{G D P}}=\gamma_{1}+\gamma_{2} \frac{G D P_{i t}}{\text { Norm }_{i t}}
$$

And the coefficients $\gamma_{1}$ and $\gamma_{2}$ test the relevance of the normalizations $\frac{F X I}{G D P}$ and $\frac{F X I}{\text { NormJ re- }}$ spectively. Results (Table 5) do not point to a preferred metric, indicating also that the effect

\begin{tabular}{|c|c|c|c|c|}
\hline & (1) & $(2)$ & $(3)$ & (4) \\
\hline FXI/GDP (instrumented) & $\begin{array}{c}0.226 \\
(1.167)\end{array}$ & $\begin{array}{c}0.004 \\
(1.673)\end{array}$ & $\begin{array}{c}-1.851^{*} \\
(1.116)\end{array}$ & $\begin{array}{l}-1.131 \\
(0.806)\end{array}$ \\
\hline FXI/M2 (instrumented) & $\begin{array}{l}-0.528 \\
(0.342)\end{array}$ & & & \\
\hline FXI/Financial sector size (IFS, instrumented) & & $\begin{array}{l}-0.790 \\
(0.838)\end{array}$ & & \\
\hline FXI/Financial sector size (WB, instrumented) & & & $\begin{array}{c}0.031 \\
(0.395)\end{array}$ & \\
\hline FXI/Market size (instrumented) & & & & $\begin{array}{l}-0.058 \\
(0.058)\end{array}$ \\
\hline Dependent variable (lagged) & $\begin{array}{c}0.964^{* * *} \\
(0.004)\end{array}$ & $\begin{array}{c}0.965^{* * *} \\
(0.004)\end{array}$ & $\begin{array}{c}0.967^{* * * *} \\
(0.004)\end{array}$ & $\begin{array}{c}0.965^{* * *} \\
(0.004)\end{array}$ \\
\hline VIX & $\begin{array}{c}-0.044^{* * *} \\
(0.005)\end{array}$ & $\begin{array}{c}-0.041^{* * *} \\
(0.006)\end{array}$ & $\begin{array}{c}-0.050^{* * *} \\
(0.006)\end{array}$ & $\begin{array}{c}-0.047^{* * *} \\
(0.006)\end{array}$ \\
\hline Interest rate (differential) & $\begin{array}{c}0.088 \\
(0.119)\end{array}$ & $\begin{array}{c}0.006 \\
(0.125)\end{array}$ & $\begin{array}{l}-0.030 \\
(0.125)\end{array}$ & $\begin{array}{c}0.052 \\
(0.131)\end{array}$ \\
\hline Commodity prices & Yes & Yes & Yes & Yes \\
\hline Country fixed effects & Yes & Yes & Yes & Yes \\
\hline Observations & 9053 & 8741 & 8424 & 8515 \\
\hline Countries & 52 & 52 & 48 & 50 \\
\hline$R^{2}$ & 0.98 & 0.98 & 0.98 & 0.98 \\
\hline $\mathrm{J}$ p-value & 0.32 & 0.23 & 0.22 & 0.23 \\
\hline Stock \& Yogo stat & 2.89 & 1.92 & 2.96 & 1.41 \\
\hline
\end{tabular}
of interventions on the exchange rate is not significantly influenced by the depth of the domestic financial system. ${ }^{25}$

Table 5: Testing different normalizations, second stage

Standard errors robust to autocorrelation and heteroskedasticity in parentheses

${ }^{*} p<0.10,{ }^{* *} p<0.05,{ }^{* * *} p<0.01$

\footnotetext{
${ }^{25}$ This result is consistent with recent evidence of FXI in countries with large financial systems (e.g. Switzerland, Japan).
} 


\section{Instrumentation}

We conduct two exercises to assess the robustness of our results with respect to the instrumentation strategy. First, we test whether results are driven by any single instrument, by excluding one instrument at a time. Tables 6 and 7 show that (first and second stage) results are robust to dropping any single instrument. While the strength of the instrumentation comes in part from the change in M2 (as indicated by the weakening of the Stock and Yogo test) the sign and magnitude of the coefficient of FXI in the second stage remains close to the baseline, as well as statistically significant. Results also hold even when both measures related to M2 are excluded from the specification, indicating that the results do not depend on having a measure of broad money in the set of instruments. The coefficient of interest varies between -1.4 and -1.8 , which is very close to the baseline specification.

Table 6: Instrumentation, second stage

\begin{tabular}{|c|c|c|c|c|c|c|}
\hline & (1) & $(2)$ & $(3)$ & $\begin{array}{c}(4) \\
\text { Dropping from inst }\end{array}$ & $\begin{array}{r}(5) \\
\text { iments: }\end{array}$ & (6) \\
\hline & Baseline & $\begin{array}{l}\text { Import } \\
\text { coverage }\end{array}$ & $\begin{array}{l}\text { Broad money } \\
\text { coverage }\end{array}$ & $\begin{array}{l}\text { Dep. Dollarization } \\
\times \text { VIX }\end{array}$ & $\begin{array}{l}\text { Change in } \\
\text { M2/GDP }\end{array}$ & $\begin{array}{l}\text { Change in M2 \& } \\
\text { Broad money coverage }\end{array}$ \\
\hline FXI/GDP (instrumented) & $\begin{array}{c}-1.430^{* * *} \\
(0.326)\end{array}$ & $\begin{array}{c}-1.468^{* * *} \\
(0.349)\end{array}$ & $\begin{array}{c}-1.424^{* * *} \\
(0.326)\end{array}$ & $\begin{array}{c}-1.395^{* * *} \\
(0.334)\end{array}$ & $\begin{array}{c}-1.771^{* *} \\
(0.721)\end{array}$ & $\begin{array}{c}-1.753^{* *} \\
(0.718)\end{array}$ \\
\hline Dependent variable (lagged) & $\begin{array}{c}0.966^{* * *} \\
(0.004)\end{array}$ & $\begin{array}{c}0.966^{* * *} \\
(0.004)\end{array}$ & $\begin{array}{c}0.966^{* * *} \\
(0.003)\end{array}$ & $\begin{array}{c}0.966^{* * *} \\
(0.004)\end{array}$ & $\begin{array}{c}0.965^{* * *} \\
(0.004)\end{array}$ & $\begin{array}{c}0.965^{* * *} \\
(0.004)\end{array}$ \\
\hline VIX & $\begin{array}{c}-0.043^{* * *} \\
(0.005)\end{array}$ & $\begin{array}{c}-0.043^{* * *} \\
(0.006)\end{array}$ & $\begin{array}{c}-0.042^{* * *} \\
(0.005)\end{array}$ & $\begin{array}{l}-0.042^{* * *} \\
(0.005)\end{array}$ & $\begin{array}{c}-0.045^{* * *} \\
(0.008)\end{array}$ & $\begin{array}{c}-0.045^{* * *} \\
(0.008)\end{array}$ \\
\hline Interest rate (differential) & $\begin{array}{c}0.014 \\
(0.117)\end{array}$ & $\begin{array}{c}0.011 \\
(0.118)\end{array}$ & $\begin{array}{c}0.015 \\
(0.117)\end{array}$ & $\begin{array}{c}0.018 \\
(0.116)\end{array}$ & $\begin{array}{l}-0.017 \\
(0.131)\end{array}$ & $\begin{array}{l}-0.015 \\
(0.131)\end{array}$ \\
\hline Commodity prices & Yes & Yes & Yes & Yes & Yes & Yes \\
\hline Country fixed effects & Yes & Yes & Yes & Yes & Yes & Yes \\
\hline Observations & 9149 & 9149 & 9149 & 9149 & 9149 & 9149 \\
\hline Countries & 52 & 52 & 52 & 52 & 52 & 52 \\
\hline$R^{2}$ & 0.98 & 0.98 & 0.98 & 0.98 & 0.98 & 0.98 \\
\hline $\mathrm{J}$ p-value & 0.39 & 0.59 & 0.35 & 0.26 & 0.35 & 0.29 \\
\hline Stock \& Yogo stat & 19.42 & 28.00 & 24.01 & 22.38 & 5.07 & 6.48 \\
\hline
\end{tabular}

Standard errors robust to autocorrelation and heteroskedasticity in parentheses

${ }^{*} p<0.10,{ }^{* *} p<0.05,{ }^{* * *} p<0.01$

Second, we employ only one instrument at a time (dropping all others). As shown in Table 8, the significance of the second stage FXI coefficient falls, to different degrees - and so do the instrumentation tests - but the magnitude of estimated FXI coefficient remains very close to the baseline results. Table 9 shows the strength of the relationship between FXI and each of the instruments. 
Table 7: Instrumentation, first stage

\begin{tabular}{|c|c|c|c|c|c|c|}
\hline & (1) & $(2)$ & $(3)$ & $(4)$ & $(5)$ & $(6)$ \\
\hline Exchange rate (lagged) & $\begin{array}{c}-0.003^{* *} \\
(0.001)\end{array}$ & $\begin{array}{c}-0.003^{* * *} \\
(0.001)\end{array}$ & $\begin{array}{c}-0.003^{* *} \\
(0.001)\end{array}$ & $\begin{array}{c}-0.003^{* * *} \\
(0.001)\end{array}$ & $\begin{array}{c}-0.003^{* *} \\
(0.001)\end{array}$ & $\begin{array}{c}-0.003^{* *} \\
(0.001)\end{array}$ \\
\hline VIX & $\begin{array}{c}-0.005^{* *} \\
(0.002)\end{array}$ & $\begin{array}{c}-0.005^{* *} \\
(0.002)\end{array}$ & $\begin{array}{c}-0.005^{* *} \\
(0.002)\end{array}$ & $\begin{array}{c}-0.007^{* * *} \\
(0.002)\end{array}$ & $\begin{array}{c}-0.005^{* *} \\
(0.002)\end{array}$ & $\begin{array}{c}-0.005^{* *} \\
(0.002)\end{array}$ \\
\hline Interest rate (differential) & $\begin{array}{c}-0.095^{* *} \\
(0.037)\end{array}$ & $\begin{array}{c}-0.091^{* *} \\
(0.038)\end{array}$ & $\begin{array}{c}-0.095^{* *} \\
(0.039)\end{array}$ & $\begin{array}{c}-0.105^{* * *} \\
(0.039)\end{array}$ & $\begin{array}{c}-0.097^{* *} \\
(0.039)\end{array}$ & $\begin{array}{r}-0.097^{* *} \\
(0.040)\end{array}$ \\
\hline Change in M2/GDP & $\begin{array}{c}0.104^{* * *} \\
(0.022)\end{array}$ & $\begin{array}{c}0.104^{* * *} \\
(0.022)\end{array}$ & $\begin{array}{c}0.104^{* * *} \\
(0.022)\end{array}$ & $\begin{array}{c}0.105^{* * *} \\
(0.022)\end{array}$ & & \\
\hline Financial dollarization $\times$ VIX & $\begin{array}{c}-0.011^{* *} \\
(0.005)\end{array}$ & $\begin{array}{c}-0.010^{* *} \\
(0.005)\end{array}$ & $\begin{array}{c}-0.011^{* *} \\
(0.005)\end{array}$ & & $\begin{array}{c}-0.013^{* *} \\
(0.005)\end{array}$ & $\begin{array}{c}-0.013^{* *} \\
(0.005)\end{array}$ \\
\hline Import coverage (lagged) & $\begin{array}{c}-0.252^{*} \\
(0.142)\end{array}$ & & $\begin{array}{c}-0.262^{* *} \\
(0.113)\end{array}$ & $\begin{array}{c}-0.243^{*} \\
(0.140)\end{array}$ & $\begin{array}{c}-0.242^{*} \\
(0.144)\end{array}$ & $\begin{array}{r}-0.249^{* *} \\
(0.116)\end{array}$ \\
\hline Low import coverage (lagged) & $\begin{array}{c}0.035 \\
(0.042)\end{array}$ & & $\begin{array}{c}0.037 \\
(0.045)\end{array}$ & $\begin{array}{c}0.041 \\
(0.041)\end{array}$ & $\begin{array}{c}0.038 \\
(0.044)\end{array}$ & $\begin{array}{c}0.039 \\
(0.047)\end{array}$ \\
\hline Broad money coverage (lagged) & $\begin{array}{l}-0.021 \\
(0.145)\end{array}$ & $\begin{array}{c}-0.219^{*} \\
(0.130)\end{array}$ & & $\begin{array}{l}-0.042 \\
(0.140)\end{array}$ & $\begin{array}{l}-0.015 \\
(0.143)\end{array}$ & \\
\hline Commodity prices & Yes & Yes & Yes & Yes & Yes & Yes \\
\hline Country fixed effects & Yes & Yes & Yes & Yes & Yes & Yes \\
\hline Observations & 9149 & 9149 & 9149 & 9149 & 9149 & 9149 \\
\hline Countries & 52 & 52 & 52 & 52 & 52 & 52 \\
\hline$R^{2}$ & 0.10 & 0.10 & 0.10 & 0.10 & 0.09 & 0.09 \\
\hline F stat & 8.18 & 12.22 & 10.22 & 8.01 & 3.84 & 5.16 \\
\hline F p-value & 0.00 & 0.00 & 0.00 & 0.00 & 0.01 & 0.00 \\
\hline
\end{tabular}

Standard errors robust to autocorrelation and heteroskedasticity in parentheses

${ }^{*} p<0.10,{ }^{* *} p<0.05,{ }^{* * *} p<0.01$

Table 8: Using individual instruments, second stage

\begin{tabular}{lcccc}
\multicolumn{5}{c}{ Dependent variable: real bilateral exchange rate } \\
\hline \hline Instrumenting only with: & $\begin{array}{c}(1) \\
\text { Change in } \\
\text { M2/GDP }\end{array}$ & $\begin{array}{c}(2) \\
\text { Dep. } \begin{array}{c}\text { Dollarization } \\
\times \text { VIX }\end{array}\end{array}$ & $\begin{array}{c}(3) \\
\text { Import } \\
\text { coverage }\end{array}$ & $\begin{array}{c}(4) \\
\text { Broad money } \\
\text { coverage }\end{array}$ \\
\hline FXI/GDP (instrumented) & $-1.310^{* * *}$ & -1.995 & $-1.614^{* *}$ & $-2.454^{*}$ \\
& $(0.375)$ & $(1.389)$ & $(0.803)$ & $(1.365)$ \\
Dependent variable (lagged) & $0.967^{* * *}$ & $0.964^{* * *}$ & $0.966^{* * *}$ & $0.962^{* * *}$ \\
& $(0.004)$ & $(0.006)$ & $(0.005)$ & $(0.007)$ \\
VIX & $-0.042^{* * *}$ & $-0.047^{* * *}$ & $-0.044^{* * *}$ & $-0.051^{* * *}$ \\
& $(0.006)$ & $(0.013)$ & $(0.008)$ & $(0.012)$ \\
Interest rate (differential) & 0.025 & -0.037 & -0.002 & -0.079 \\
& $(0.118)$ & $(0.173)$ & $(0.133)$ & $(0.172)$ \\
\hline Commodity prices & Yes & Yes & Yes & Yes \\
Country fixed effects & Yes & Yes & Yes & Yes \\
\hline Observations & 9149 & 9149 & 9149 & 9149 \\
Countries & 52 & 52 & 52 & 52 \\
$R^{2}$ & 0.98 & 0.98 & 0.98 & 0.97 \\
J p-value & & & 0.11 & 5.69 \\
Stock \& Yogo stat & 68.86 & 8.48 & 6.98 & 5 \\
\hline \hline
\end{tabular}

Standard errors robust to autocorrelation and heteroskedasticity in parentheses

${ }^{*} p<0.10,{ }^{* *} p<0.05,{ }^{* * *} p<0.01$ 
Table 9: Using individual instruments, first stage

Dependent variable: foreign exchange intervention (\% GDP)

\begin{tabular}{|c|c|c|c|c|}
\hline & $(1)$ & $(2)$ & $(3)$ & $(4)$ \\
\hline Exchange rate (lagged) & $\begin{array}{c}-0.004^{* * *} \\
(0.001)\end{array}$ & $\begin{array}{c}-0.004^{* * *} \\
(0.001)\end{array}$ & $\begin{array}{c}-0.003^{* *} \\
(0.001)\end{array}$ & $\begin{array}{c}-0.003^{* * *} \\
(0.001)\end{array}$ \\
\hline VIX & $\begin{array}{c}-0.008^{* * *} \\
(0.002)\end{array}$ & $\begin{array}{c}-0.005^{* *} \\
(0.002)\end{array}$ & $\begin{array}{c}-0.008^{* * *} \\
(0.002)\end{array}$ & $\begin{array}{c}-0.008^{* * *} \\
(0.002)\end{array}$ \\
\hline Interest rate (differential) & $\begin{array}{c}-0.087^{* *} \\
(0.042)\end{array}$ & $\begin{array}{c}-0.080^{*} \\
(0.041)\end{array}$ & $\begin{array}{c}-0.107^{* *} \\
(0.041)\end{array}$ & $\begin{array}{c}-0.104^{* *} \\
(0.040)\end{array}$ \\
\hline Change in $\mathrm{M} 2 / \mathrm{GDP}$ & $\begin{array}{c}0.104^{* * *} \\
(0.022)\end{array}$ & & & \\
\hline Financial dollarization $\times$ VIX & & $\begin{array}{c}-0.014^{* * *} \\
(0.005)\end{array}$ & & \\
\hline Import coverage (lagged) & & & $\begin{array}{c}-0.251^{* *} \\
(0.116)\end{array}$ & \\
\hline Low import coverage (lagged) & & & $\begin{array}{c}0.047 \\
(0.046)\end{array}$ & \\
\hline Broad money coverage (lagged) & & & & $\begin{array}{c}-0.227^{*} \\
(0.125)\end{array}$ \\
\hline Commodity prices & Yes & Yes & Yes & Yes \\
\hline Country fixed effects & Yes & Yes & Yes & Yes \\
\hline Observations & 9149 & 9149 & 9149 & 9149 \\
\hline Countries & 52 & 52 & 52 & 52 \\
\hline$R^{2}$ & 0.10 & 0.09 & 0.09 & 0.09 \\
\hline F stat & 22.51 & 8.38 & 4.65 & 3.30 \\
\hline F p-value & 0.00 & 0.01 & 0.01 & 0.08 \\
\hline
\end{tabular}

Standard errors robust to autocorrelation and heteroskedasticity in parentheses

${ }^{*} p<0.10,{ }^{* *} p<0.05,{ }^{* * *} p<0.01$

\section{Exchange rate pegs}

The baseline estimations include all types of exchange rate regimes (except those classified as de facto free falling or dual exchange rates by Ilzetzki et al. (2010); and countries without their own currency). To confirm that our results are not driven by currencies under pegged regimes, we restrict the sample excluding de-jure regimes with some form of exchange rate targeting, according to three different criteria:

i. excluding de-jure pegs and crawling pegs;

ii. excluding the latter and 'narrow crawling band' regimes (narrower than or equal to $+/-2 \%$ ); and

iii. excluding the latter two and 'wide crawling band'regimes (narrower than or equal to $+/-5 \%$ ).

Results, presented in Appendix Table A5, are very similar to those of the baseline estimation, with FXI coefficients of somewhat larger absolute magnitude. 
The case for excluding de-facto exchange rate targeting regimes is less clear, since these can be thought of as instances where there is clear evidence of effectiveness of FXI. Still, to test the robustness of our results, we restrict the sample in the same way as above, but by excluding cases of de-facto peg exchange rate regimes. Results, also presented in Appendix Table A5, are broadly similar to the baseline.

\section{Other policy responses}

Policy measures other than FXI can also influence or affect the impact of FXI on the exchange rate. Capital control measures and changes in the monetary policy interest rate are most prominent at high frequency. Adequately controlling for these is key to ensure our estimates on FXI are unbiased.

Capital controls. The presence of capital flow restrictions gives rise to two issues. First, contemporaneous changes in those restrictions could lead to omitted variable bias if not controlled for. Ideally, the estimation should include a measure of changes in such restrictions. However, existing capital control indices are only available (for a large set of countries) at an annual frequency, thus preventing a proper identification of the timing of the measures at monthly frequency. To bypass this obstacle, we exclude from the sample country/year observations for which a change in the Quinn and Toyoda index of capital controls is identified at an annual frequency. ${ }^{26}$ The sample is reduced by about 7 percent, and results (Appendix Table A6, columns 1 and 2) do not change significantly. The coefficient of interest is slightly smaller in absolute value, suggesting that there is some use of capital flow measures contemporaneously to FXI in the baseline sample. Second, assuming homogeneous coefficient for the control variables may affect the FXI coefficient estimate if such effects vary across countries. Capital flow restrictions could be the cause of such heterogeneity. To allow for such heterogeneity related to capital controls, we modify the baseline specification by including interactions of the key external financial variables (that would normally drive capital flows) with the lagged index of capital controls. ${ }^{27}$ We find results similar to the baseline specification (Appendix Table A6, columns 3 and 4). ${ }^{28}$

Interest rate changes. One somewhat surprising result in the baseline specification relates to the coefficient of the interest rate differential in the second stage, which is quite small and nonsignificant. Two main issues could affect the result. First, the interest rate may be endogenous to exchange rate movements, if countries in our sample used the interest rate as a tool to 'defend' the exchange rate. Such endogeneity would tend to hide the true effect of interest rate shocks on the exchange rate. To explore this, we estimate an alternative model where we also instrument the interest rate differential by adding an extra equation in the first stage for the interest rate. ${ }^{29}$

\footnotetext{
${ }^{26}$ This criterion should suffice to exclude within year variations. There is still the possibility that capital flow measures are taken and reverted within the year, and so would not show in the annual values. However, countries rarely use capital control in such high frequency basis. See, for example, Fernández et al. (2013).

${ }^{27}$ As before, we employ the Quinn and Toyoda index, which varies between 0 and 100 (100 being more open).

${ }^{28}$ The possible complementarity of FXI and capital controls is also a relevant aspect, although beyond the scope of this paper. See Annex 2 for a related discussion.

${ }^{29}$ Whether the set of instruments used for FXI is appropriate for the interest rate is certainly debatable, although the instrumentation tests are passed. Arguably, instrumenting the interest rate properly with the aim of identifying its effect on exchange rates would likely entail searching for exogenous reasons for moving the policy rate, beyond those found for FXI. However, our interest does not lie in the interest rate coefficient. Our goal is simply to ensure that the possible correlation between interest rate changes and FXI does not lead to a bias in the coefficient of the latter. That is, there might be other sources of exogenous interest rate movements, but provided they do not drive exogenous FXI movements our estimation should not suffer from collinearity between interest rates and FXI. This justifies the use of the same set of instruments for both variables.
} 
Second, domestic and foreign interest rates may have asymmetric effects on the exchange rate. To allow for this possibility, we introduce these two interest rates separately in the model. These variations deliver mixed results (Appendix Table A7) regarding the estimated effect of interest rate changes on the exchange rate, but in all cases, the coefficient of FXI in the second stage remains basically unaltered. ${ }^{30}$

\section{Measures of FXI}

So far, we have relied on the change in the central bank NFA position as a proxy for FXI. While this is consistent with other studies, this measure is polluted by factors other than FXI that affect the NFA position, like valuation effects and income flows generated by assets and liabilities. Thus, we conduct a series of robustness checks on our proxy for FXI:

i. Excluding observations with small absolute values (10\% smallest) of FXI, as these may be particularly affected by valuation effects and income flows.

ii. Using a more refined proxy of FXI that adjusts for estimates of valuation effects and income flows. A detailed description of the methodology is available in Annex 1.

iii. Including off-balance sheet operations in our proxy of FXI in order to capture the increasing use of non-spot instruments (i.e. FX derivatives). See also Annex 1.

iv. Using changes in gross international reserves (rather than relaying on a net concept).

We find that results are very stable, reflecting the fact that the different measures of FXI are highly correlated (see Appendix Tables A8 and A9).

\subsection{Other robustness checks}

We conduct a number of additional robustness checks (reported in Appendix Table A10), including:

i. Exploring alternative measures of exogenous financial shocks, substituting the VIX by the EMBI Global sovereign spread or our measure of EPFR flows to other countries. These alternative variables are used both as controls and as instruments interacted with deposits dollarization.

ii. Allowing for country-specific coefficients for VIX, interest rate, and both.

iii. Changing the treatment of outliers and the sample period. This includes dropping $2 \%$ top and bottom outliers; winsorizing the data (1\% and $2 \%$ ); dropping the crisis period (from June 2008 to May 2009); dropping the Asian crisis period; and dropping the largest 5\% and $10 \%$ FXI/GDP observations.

iv. Considering possible structural breaks related to changes in monetary policy regimes. This includes restricting the sample period to 2003-13 (as most countries consolidated their current regimes in the early 2000s); and restricting the sample only to inflation targeting (IT) countries/periods.

\footnotetext{
${ }^{30}$ For the instrumentation of the interest rate differential, Findol $\times V I X$ is significant and positive in the first stage, suggesting that indeed, in dollarized economies, the interest rate is used as a tool to stabilize the value of the domestic currency in response to external financial (VIX) shocks.
} 
v. Adding a measure of the real effective exchange rate gap, defined by deviations from an HP filter.

vi. Finally, estimating a model in first differences out of possible concerns about stationarity. The estimated coefficient for FXI is still negative and statistically significant, although smaller in absolute magnitude. The latter is not surprising since this specification differs from the benchmark in its assumptions about exchange rate dynamics. In the baseline, the introduction of lagged exchange rate level allows for a gradual mean reversion of shocks, while the first differences specification imposes the assumption that shocks have permanent effects. In the next section, we study the persistence of FXI effects more in depth.

Overall, results display very stable estimates for the effect of FXI on the exchange rate, with magnitudes consistent with those of the baseline specification.

\section{Extensions}

\subsection{Dynamic Effects}

So far, we have focused on the contemporaneous impact of FXI on the exchange rate. And, while the baseline specification allows for some persistent effects through the autoregressive term, different shocks (including FXI) may have different degrees of persistence. In this section we extend our analysis to study this aspect, the understanding of which is paramount to the policy maker. Specifically, lagged values of FXI are added to our benchmark specification. We use up to three lags as coefficients on further lags were not significant. Contemporaneous FXI is instrumented as before, while lags of FXI are included in both the first and second stages as controls as these are exogenous to the current exchange rate. Figure 3 displays the implied dynamics of a FXI (FX purchase) of 1 percent of GDP for the different exchange rate measures (corresponding to columns 1-3 in Appendix Table A11). The immediate impact of FXI on the exchange rate remains similar to our benchmark specification. Effects are relatively persistent, and relatively more so for the nominal than for the real exchange rate. The coefficients of the three FXI lags are positive, meaning that the impact of FXI on the exchange rate decreases over time and that this decay is faster than that of an average shock (captured by the auto-regressive coefficient of the exchange rate). The half-life of FXI shocks (bottom row of Appendix Table A11) ranges between 12 and 23 months, displaying the highest values for the nominal bilateral exchange rate, and lowest for the real effective exchange rate. This result suggests that FXI may be more effective in affecting nominal than real variables, as one would expect.

These half-life estimates remain below those of a general shock to the exchange rate, which lie between 18 and 29 months according to our baseline estimates. The latter are broadly consistent with findings of previous studies, which ranged between 2 and 7 years, depending on the approach. ${ }^{31}$ A number of additional robustness checks (see Appendix Table A12) confirm these results. ${ }^{32}$

\subsection{Asymmetric effects}

Finally, we study whether FXI effects are asymmetric, i.e. whether positive interventions (FX purchases) are more/less effective than negative ones (FX sales). A priori, one can think of two

\footnotetext{
${ }^{31}$ Frankel and Rose (1996) find a half-life of 4 years for a panel of 150 countries; while Cheung and Lai (2000) obtain a half-life of 2-5 years for advanced countries, and below 3 years for emerging economies. More recently, Chortareas and Kapetanios (2013) suggest a new definition of the half-life, and obtain estimates ranging from 1 to 2 years.

${ }^{32}$ Without the outlier treatment, the effect of FXI is in general stronger, but also less persistent, reflecting a combination of smaller autoregressive coefficients and higher coefficients for the lagged FXI regressors.
} 
Figure 3: Impulse response functions of nominal, real and real effective exchange rates

(a) NER

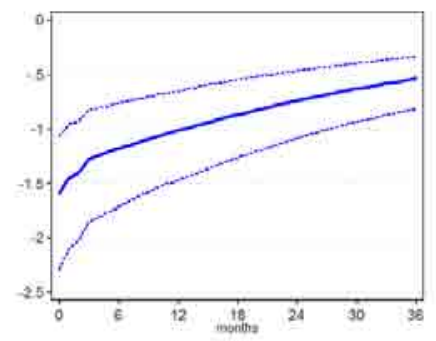

(b) RER



(c) REER

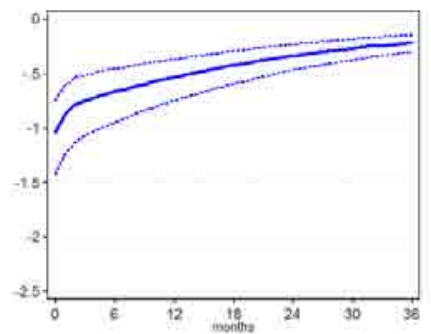

Dashed lines show bootstrapped confidence intervals.

different reasons for possible asymmetric effects, which go in opposite directions:

i. On the one hand, a lower bound on the amount of reserves may constrain the central bank's ability to defend a specific exchange rate level. As agents anticipate this, FX sales may have limited effects on the exchange rate.

ii. On the other hand, while the central bank balance sheet can be expanded unlimitedly through positive FXI (FX purchases), larger FX positions tend to entail a quasi fiscal cost and so FXI may not be sustained indefinitely. ${ }^{33}$ Expectations about the later could, again, render FXI ineffective.

We examine possible asymmetric effects by enriching the specification to allow for a differentiated effect. Specifically:

$$
\begin{aligned}
\log \left(E R_{i t}\right) & =\alpha+\beta \log \left(E R_{i t-1}\right)+\gamma_{1} \widehat{F X I}_{i t}+\gamma_{2} \widehat{F X I_{i t}^{P O}}+\boldsymbol{\delta}^{\prime} \mathbf{X}_{i t}+\eta_{i}+\epsilon_{i t} \\
F X I_{i t} & =a_{1}+b_{1} \log \left(E R_{i t-1}\right)+\mathbf{c}_{1}^{\prime} \mathbf{Z}_{i t}+\mathbf{e}_{1}^{\prime}\left[\mathbf{Z}_{i t} \times \mathbb{I}\left(\widehat{F X I}_{i t}>0\right)\right]+\mathbf{d}_{1}^{\prime} \mathbf{X}_{i t}+u_{i}+v_{i t} \\
\widehat{F X I_{i t}^{P O} S} & \equiv \widehat{F X I}_{i t} \times \mathbb{I}\left(\widehat{F X I}_{i t}>0\right)
\end{aligned}
$$

Where the innovation relative to the baseline is that positive interventions $\left(F X I_{i t}^{P O S}\right)$ are instrumented separately. Our interest lies in the sign of the coefficient $\gamma_{2}$, which indicates whether positive interventions are more $\left(\gamma_{2}<0\right)$ or less effective $\left(\gamma_{2}>0\right)$ than negative ones.

As is shown in Table 10, we find no evidence of an asymmetric effect in either direction, suggesting that positive and negative FXI are equally effective.

\section{Conclusions}

We study the effect of FXI on the level of the exchange rate, using an instrumental-variables panel setting in a large sample of countries and time. We find robust evidence that intervention affects the level of the exchange rate in a meaningful way. A purchase of foreign currency of 1 percentage point of GDP causes a depreciation of the nominal and real exchange rate in the ranges

\footnotetext{
${ }^{33}$ See Adler and Mano (2015) for a documentation of the quasi fiscal costs of carrying out FXI.
} 
of [1.7-2.0] percent and [1.4-1.7] percent respectively. Effects are found to be quite persistent, pointing to a half-life in the range of [12-23] months, depending on the specification. Finally, positive and negative interventions appear to be equally effective, suggesting that FXI is a useful policy tool both when facing appreciation and depreciation pressures. Overall, these results indicate that FXI is a valid policy instrument for macroeconomic management, and its merits should be judged less on the basis of whether it is effective or not, and more on its relative costs and benefits vis-a-vis other instruments. 
Table 10: Asymmetric effects: Second stage

\begin{tabular}{|c|c|c|c|c|c|c|c|c|c|c|c|c|}
\hline \multicolumn{13}{|c|}{ Dependant variable: exchange rate } \\
\hline \multirow[b]{3}{*}{ Exchange rate definition: } & $(1)$ & $\overline{(2)}$ & $(3)$ & \multirow{2}{*}{\multicolumn{3}{|c|}{$\begin{array}{cc}(5) & (5) \\
\text { Excluding de jure pegs }\end{array}$}} & \multirow{2}{*}{\multicolumn{3}{|c|}{$\begin{array}{l}\text { (7) } \\
\text { Including de jure pegs }\end{array}$}} & \multirow{2}{*}{\multicolumn{3}{|c|}{ Excluding de jure pegs }} \\
\hline & \multicolumn{3}{|c|}{ Including de jure pegs } & & & & & & & & & \\
\hline & Nominal & Real & Real effective & \multicolumn{3}{|c|}{$\begin{array}{l}\text { Excluding de jure pegs } \\
\text { Nominal } \quad \text { Real } \quad \text { Real effective }\end{array}$} & \multicolumn{3}{|c|}{$\begin{array}{l}\text { Nominal Real Real effective } \\
\text { Real Re }\end{array}$} & \multicolumn{2}{|c|}{ Nominal Real } & \multirow{2}{*}{$\begin{array}{c}\text { Real effective } \\
-1.182 \\
(1.264)\end{array}$} \\
\hline FXI/GDP (instrumented) & $\begin{array}{l}-1.224 \\
(0.783)\end{array}$ & $\begin{array}{l}-1.392 \\
(0.905)\end{array}$ & $\begin{array}{c}-2.523^{* * *} \\
(0.772)\end{array}$ & $\begin{array}{l}-0.914 \\
(1.085)\end{array}$ & $\begin{array}{l}-1.016 \\
(1.246)\end{array}$ & $\begin{array}{c}-2.438^{* *} \\
(1.232)\end{array}$ & $\begin{array}{l}-1.370 \\
(0.953)\end{array}$ & $\begin{array}{l}-1.398 \\
(0.909)\end{array}$ & $\begin{array}{l}-1.638^{*} \\
(0.925)\end{array}$ & $\begin{array}{l}-0.572 \\
(1.128)\end{array}$ & $\begin{array}{l}-0.403 \\
(1.116)\end{array}$ & \\
\hline Positive FXI/GDP & $\begin{array}{l}-0.219 \\
(0.557)\end{array}$ & $\begin{array}{l}0.180 \\
(0.769)\end{array}$ & $\begin{array}{c}1.720^{* * *} \\
(0.652)\end{array}$ & $\begin{array}{l}-0.651 \\
(0.855)\end{array}$ & $\begin{array}{l}-0.528 \\
(1.083)\end{array}$ & $\begin{array}{l}1.366 \\
(1.100)\end{array}$ & $\begin{array}{l}-0.287 \\
(0.650)\end{array}$ & $\begin{array}{l}-0.023 \\
(0.680)\end{array}$ & $\begin{array}{l}0.439 \\
(0.774)\end{array}$ & $\begin{array}{l}-1.102 \\
(0.870)\end{array}$ & $\begin{array}{l}-1.136 \\
(0.952)\end{array}$ & $\begin{array}{c}0.014 \\
(1.064)\end{array}$ \\
\hline Dependant variable (lagged) & $\begin{array}{c}0.977^{* * *} \\
(0.005)\end{array}$ & $\begin{array}{c}0.968^{* * *} \\
(0.005)\end{array}$ & $\begin{array}{c}0.963^{* * *} \\
(0.005)\end{array}$ & $\begin{array}{c}0.972^{* * *} \\
(0.004)\end{array}$ & $\begin{array}{c}0.962^{* * *} \\
(0.005)\end{array}$ & $\begin{array}{c}0.961^{* * *} \\
(0.007)\end{array}$ & $\begin{array}{c}0.974^{* * *} \\
(0.006)\end{array}$ & $\begin{array}{c}0.953^{* * *} \\
(0.007)\end{array}$ & $\begin{array}{c}0.959^{* * *} \\
(0.006)\end{array}$ & $\begin{array}{c}0.968^{* * *} \\
(0.005)\end{array}$ & $\begin{array}{c}0.946^{* * *} \\
(0.006)\end{array}$ & $\begin{array}{c}0.958^{* * *} \\
(0.007)\end{array}$ \\
\hline VIX & $\begin{array}{c}-0.052^{* * *} \\
(0.008)\end{array}$ & $\begin{array}{c}-0.038^{* * *} \\
(0.007)\end{array}$ & $\begin{array}{c}-0.012^{*} \\
(0.007)\end{array}$ & $\begin{array}{c}-0.054^{* * *} \\
(0.008)\end{array}$ & $\begin{array}{c}-0.041^{* * *} \\
(0.007)\end{array}$ & $\begin{array}{c}-0.018^{* *} \\
(0.008)\end{array}$ & $\begin{array}{c}-0.057^{* * *} \\
(0.009)\end{array}$ & $\begin{array}{c}-0.045^{* * *} \\
(0.008)\end{array}$ & $\begin{array}{c}-0.019^{* *} \\
(0.008)\end{array}$ & $\begin{array}{l}-0.056^{* * *} \\
(0.009)\end{array}$ & $\begin{array}{l}-0.045^{* * *} \\
(0.008)\end{array}$ & $\begin{array}{c}-0.018^{* *} \\
(0.009)\end{array}$ \\
\hline Interest rate (differential) & $\begin{array}{l}-0.179 \\
(0.184)\end{array}$ & $\begin{array}{c}0.065 \\
(0.171)\end{array}$ & $\begin{array}{c}0.223 \\
(0.165)\end{array}$ & $\begin{array}{l}-0.237 \\
(0.220)\end{array}$ & $\begin{array}{c}0.058 \\
(0.195)\end{array}$ & $\begin{array}{c}0.212 \\
(0.192)\end{array}$ & $\begin{array}{l}-0.179 \\
(0.198)\end{array}$ & $\begin{array}{c}0.175 \\
(0.179)\end{array}$ & $\begin{array}{c}0.348^{* * *} \\
(0.198)\end{array}$ & $\begin{array}{l}-0.205 \\
(0.243)\end{array}$ & $\begin{array}{c}0.170 \\
(0.194)\end{array}$ & $\begin{array}{c}0.316 \\
(0.225)\end{array}$ \\
\hline Inflation rate (differential) & $\begin{array}{c}0.053^{* * *} \\
(0.013)\end{array}$ & & & $\begin{array}{c}0.062^{* * *} \\
(0.016)\end{array}$ & & & $\begin{array}{c}0.057^{* * *} \\
(0.016)\end{array}$ & & & $\begin{array}{c}0.062^{* * *} \\
(0.018)\end{array}$ & & \\
\hline Commodity prices & Yes & Yes & Yes & Yes & Yes & Yes & Yes & Yes & Yes & Yes & Yes & Yes \\
\hline Observations & 8755 & 9149 & 9017 & 7688 & 8032 & 7589 & 7950 & 8312 & 7871 & 7083 & 7371 & 6980 \\
\hline Countries & 55 & 52 & 55 & 48 & 48 & 48 & 51 & 51 & 51 & 46 & 46 & 46 \\
\hline$R^{2}$ & 0.99 & 0.99 & 0.98 & 0.98 & 0.98 & 0.98 & 0.99 & 0.98 & 0.98 & 0.98 & 0.98 & 0.98 \\
\hline
\end{tabular}

Bootstrapped standard errors in parentheses

${ }^{*} p<0.10,{ }^{* *} p<0.05,{ }^{* * *} p<0.01$ 


\section{References}

AbenojA, Z. (2003): "Foreign Exchange Market Intervention: A Short Review of Transmission Channels and Practices," Bangko sentral review, Bangko Sentral ng Pilipinas.

Adler, G. And R. C. Mano (2015): "On the Cost of Foreign Exchange Intervention," mimeo.

Adler, G. AND C. E. Tovar (2014): "Foreign Exchange Interventions and their Impact on Exchange Rate Levels," Monetaria, 0, 1-48.

Aizenman, J. And J. Lee (2008): "Financial versus Monetary Mercantilism: Long-run View of Large International Reserves Hoarding," The World Economy, 31, 593-611.

Bayoumi, T., J. E. Gagnon, and C. Saborowski (2014): "Official Financial Flows, Capital Mobility, and Global Imbalances," IMF Working Papers 14/199, International Monetary Fund.

Blanchard, O., G. Adler, and I. Carvalho Filho (2015): "Can Foreign Exchange Intervention Stem Exchange Rate Pressures from Global Capital Flow Shocks?" mimeo.

Branson, W. H. And D. W. Henderson (1985): "The specification and influence of asset markets," in Handbook of International Economics, ed. by R. W. Jones and P. B. Kenen, Elsevier, vol. 2 of Handbook of International Economics, chap. 15, 749-805.

Canales-Kriljenko, J. I. (2003): "Foreign Exchange Intervention in Developing and Transition Economies: Results of a Survey," IMF Working Papers 03/95, International Monetary Fund.

Cheung, Y.-W. And K. S. Lai (2000): "On the purchasing power parity puzzle," Journal of International Economics, 52, 321-330.

Chinn, M. D. And H. Ito (2006): "What matters for financial development? Capital controls, institutions, and interactions," Journal of Development Economics, 81, 163-192.

Chortareas, G. And G. Kapetanios (2013): "How Puzzling Is The Ppp Puzzle? An Alternative HalfLife Measure Of Convergence To Ppp," Journal of Applied Econometrics, 28, 435-457.

Daude, C., E. Levy Yeyati, And A. Nagengast (2014): "On the Effectiveness of Exchange Rate Interventions in Emerging Markets," OECD Development Centre Working Papers 324, OECD Publishing.

DomaÇ, I. AND A. MEndozA (2004): "Is there room for foreign exchange interventions under an inflation targeting framework ? Evidence from Mexico and Turkey," Policy Research Working Paper Series 3288, The World Bank.

Dominguez, K. And J. A. Frankel (1990): Does Foreign Exchange Intervention Work?, no. 16 in Peterson Institute Press: All Books, Peterson Institute for International Economics.

Dominguez, K. M. (1990): "Market responses to coordinated central bank intervention," Carnegie-Rochester Conference Series on Public Policy, 32, 121-163.

- (1998): "Central Bank Intervention and Exchange Rate Volatility," Journal of International Money and Finance, 17, 161-190.

(2012): "Foreign reserve management during the global financial crisis," Journal of International Money and Finance, 31, 2017-2037. 
Dominguez, K. M., R. Fatum, And P. Vacek (2013): "Do Sales of Foreign Exchange Reserves Lead to Currency Appreciation?" Journal of Money, Credit and Banking, 45, 867-890.

Dominguez, K. M. And J. A. Frankel (1993a): "Does Foreign-Exchange Intervention Matter? The Portfolio Effect," American Economic Review, 83, 1356-69.

(1993b): "Foreign Exchange Intervention: An Empirical Assessment," in On Exchange Rates, ed. by J. A. Frankel, Cambridge, MA: MIT Press.

Dominquez, K. M. And P. B. Kenen (1992): "Intramarginal intervention in the EMS and the target-zone model of exchange-rate behavior," European Economic Review, 36, 1523-1532.

Fernández, A., A. Rebucci, And M. Uribe (2013): "Are Capital Controls Prudential? An Empirical Investigation," NBER Working Papers 19671, National Bureau of Economic Research, Inc.

Frankel, J. A. And A. K. Rose (1996): "A panel project on purchasing power parity: Mean reversion within and between countries," Journal of International Economics, 40, 209-224.

Gabaix, X. And M. Maggiori (2014): "International Liquidity and Exchange Rate Dynamics," NBER Working Papers 19854, National Bureau of Economic Research, Inc.

Gagnon, J. E. (2012a): "Combating Widespread Currency Manipulation," Policy Briefs PB12-19, Peterson Institute for International Economics.

(2012b): "Global Imbalances and Foreign Asset Expansion by Developing Economy Central Banks," Working Paper Series WP12-5, Peterson Institute for International Economics.

(2013): "The Elephant Hiding in the Room: Currency Intervention and Trade Imbalances," Working Paper Series WP13-2, Peterson Institute for International Economics.

Ghosh, A. R. (1992): "Is it signalling? Exchange intervention and the dollar-Deutschemark rate," Journal of International Economics, 32, 201-220.

Ghosh, A. R., J. D. Ostry, And C. G. Tsangarides (2012): "Shifting Motives: Explaining the Buildup in official Reserves in Emerging Markets Since the 1980's," IMF Working Papers 12/34, International Monetary Fund.

Guimarães Filho, R. F. And C. Karacada $\breve{G}$ (2004): "The Empirics of Foreign Exchange Intervention in Emerging Market Countries: the Cases of Mexico and Turkey," IMF Working Papers 04/123, International Monetary Fund.

Henderson, D. W. And K. Rogoff (1982): "Negative net foreign asset positions and stability in a world portfolio balance model," Journal of International Economics, 13, 85-104.

Humala, A. And G. Rodriguez (2010): "Foreign exchange intervention and exchange rate volatility in Peru," Applied Economics Letters, 17, 1485-1491.

Ilzetzki, E., C. M. Reinhart, And K. S. Rogoff (2010): "Exchange Rate Arrangements Entering the 21st Century: Which Anchor Will Hold?" mimeo.

JeAnne, O. And R. RAnCIÈRe (2011): "The Optimal Level of International Reserves For Emerging Market Countries: A New Formula and Some Applications," Economic Journal, 121, 905-930. 
Kamil, H. (2008): "Is Central Bank Intervention Effective Under Inflation Targeting Regimes? The Case of Colombia," IMF Working Papers 08/88, International Monetary Fund.

Kaminsky, G. L. And K. K. Lewis (1996): "Does foreign exchange intervention signal future monetary policy?" Journal of Monetary Economics, 37, 285-312.

Kenen, P. B. (1982): "Effects of Invertention and Sterilization in the Short Run and in the Long Run," in The International Monetary Sustem Under Flexible Exchange Rates: Global, Regional, and National: Essays in Honor of Robert Triffin, ed. by R. N. Cooper, P. B. Kenen, J. Braga de Macedo, and J. van Ypersele, Cambridge, MA: Ballinger.

Kouri, P. J. (1983): "Balance of Payments and the Foreign Exchange Market: A Dynamic Partial Equilibrium Model," in Economic Interndependence and Flexible Exchange Rates, ed. by J. S. Bhandari and B. H. Putnam, The MIT Press, chap. 5, 116-156.

Kumhof, M. (2010): "On the theory of sterilized foreign exchange intervention," Journal of Economic Dynamics and Control, 34, 1403-1420.

Lyons, R. K. (2006): The Microstructure Approach to Exchange Rates, vol. 1 of MIT Press Books, The MIT Press.

Mandeng, O. (2003): "Central Bank Foerign Exchange Market Intervention and Option Contract Specification: The Case of Colombia," IMF Working Papers 03/135, International Monetary Fund.

Menkhoff, L. (2010): "High-Frequency Analysis Of Foreign Exchange Interventions: What Do We Learn?" Journal of Economic Surveys, 24, 85-112.

(2013): "Foreign Exchange Intervention in Emerging Markets: A Survey of Empirical Studies," The World Economy, 36, 1187-1208.

Moreno, R. (2005): "Motives for intervention," in Foreign exchange market intervention in emerging markets: motives, techniques and implications, ed. by B. for International Settlements, Bank for International Settlements, vol. 24 of BIS Papers chapters, 4-18.

NEELY, C. J. (2005): "An analysis of recent studies of the effect of foreign exchange intervention," Review, 685-718.

(2008): "Central bank authorities' beliefs about foreign exchange intervention," Journal of International Money and Finance, 27, 1-25.

Obstfeld, M., J. C. Shambaugh, And A. M. Taylor (2010): "Financial Stability, the Trilemma, and International Reserves," American Economic Journal: Macroeconomics, 2, 57-94.

Pattanaik, S. and S. Sahoo (2003): "The Effectiveness of Intervention in India: An Empirical Assessment," Tech. rep.

Phillips, S., L. Catao, L. A. Ricci, R. Bems, M. Das, J. di Giovanni, D. F. Unsal, M. Castillo, J. Lee, J. Rodriguez, and M. Vargas (2013): "The External Balance Assessment (EBA) Methodology," IMF Working Papers 13/272, International Monetary Fund.

Quinn, D. P. And A. M. Toyoda (2008): "Does Capital Account Liberalization Lead to Growth?" Review of Financial Studies, 21, 1403-1449. 
Reinhart, C. M. and V. R. Reinhart (2008): "Capital Inflows and Reserve Accumulation: The Recent Evidence," NBER Working Papers 13842, National Bureau of Economic Research, Inc.

Rincón, H. AND J. Toro (2011): “Are Capital Controls and Central Bank Intervention Effective?" Tech. rep.

Sarno, L. And M. P. TAYlor (2001): "Official Intervention in the Foreign Exchange Market: Is It Effective and, If So, How Does It Work?" Journal of Economic Literature, 39, 839-868.

Schindler, M. (2009): "Measuring Financial Integration: A New Data Set," IMF Staff Papers, $56,222-238$.

Stock, J. H. And M. Yogo (2002): "Testing for Weak Instruments in Linear IV Regression," NBER Technical Working Papers 0284, National Bureau of Economic Research, Inc.

Stone, M. R., W. C. Walker, And Y. Yasui (2009): "From Lombard Street to Avenida Paulista: Foreign Exchange Liquidity Easing in Brazil in Response to the Global Shock of 200809," IMF Working Papers 09/259, International Monetary Fund.

TAPiA, M. And A. Tokman (2004): "Effects of Foreign Exchange Intervention under Public Information: The Chilean Case," Journal of Lacea Economia, 4:2, 215-256.

Yeyati, E. L. (2006): "Financial dollarization: evaluating the consequences," Economic Policy, 21, 61-118. 


\section{Annex 1 Proxies for FXI}

\section{Adjusting NFA for valuation and income flows}

A general problem faced by the literature on foreign exchange intervention (FXI) is the lack of comprehensive data on such operations. Only a handful of countries, mostly advanced economies, publish actual intervention data. In absence of such data, much of the research has been conducted using changes in reserves as a proxy for FXI, although the latter measure is polluted by valuation effects as well as income flows (generated by the return on assets). Here, we follow an alternative approach aiming at adjusting reserves series for such components and thus constructing a more precise proxy for intervention.

This approach is based on Dominguez (2012), except that we focus on a net concept of reserves, as opposed to a gross concept. Define country j's foreign exchange intervention (i.e., purchases) at time $t$ as:

$$
F X I_{j, t} \equiv \Delta N F C A_{j, t}-\Delta^{v a l} N F C A_{j, t}-\Delta^{i n c} N F C A_{j, t}
$$

where $\triangle N F C A_{j, t}$ denotes changes in net foreign currency assets; $\Delta^{v a l} N F C A_{j, t}$ denotes valuation changes and $\Delta^{i n c} N F C A_{j, t}$ stands for income flows on the next foreign currency position. We estimate each of these as:

$$
\begin{aligned}
\Delta N F C A_{j, t} & =\sum_{s \in S} \Delta H_{j, t}^{s} \\
\Delta^{v a l} N F C A_{j, t} & =\sum_{s \in S}\left(P_{t}^{s}-P_{t-1}^{s}\right) H_{j, t-1}^{s} \\
\Delta^{i n c} N F C A_{j, t} & =\sum_{s \in S} i_{t-1}^{s} H_{j, t-1}^{s}
\end{aligned}
$$

where $H_{j, t}^{s}$ is the net position on a security $s$ at time $t$; $P_{t}^{s}$ denotes the market price of foreign asset $s$ at time $t$; and $i_{t}^{s}$ denotes the monthly interest rate on those securities. The price of a (zero coupon) security maturing $\mathrm{n}$ months ahead is estimated by $\left(1+i_{t}^{s}\right)^{-n}$.

The break-down of foreign currency assets into different securities is given by the IMF's Data Template on International Reserves and Foreign Currency Liquidity. The currency composition of the different securities (not provided by the template) is assumed to be uniform across the asset class, and broken down into 7 major currencies, as indicated in the Currency Composition of Official Foreign Exchange Reserves (COFER) dataset. These include: US dollar, the Australian dollar, Canadian dollar, British pound, Japanese Yen, Swiss Franc and Euro. Because the Fund's reserve template only focuses on assets, the data is complemented by using the series of central bank foreign liabilities available through IMF's International Financial Statistics. As noted by Dominguez (2012), most central banks claim to hold primarily long-term government bonds. Thus, we follow her in assuming that securities are mostly composed of 10-year bonds. Also along the lines of Dominguez (2012), 3-month interbank yields are used as proxies for returns on holdings of foreign currency and deposits. Valuation effects are zero for the latter assets. The IMF's reserve position and holdings of SDR are valued at the SDR rate, and generate income according to the SDR interest rate. Gold holdings are valued at market prices. Table 11 summarizes the information used to make this valuation and income flow adjustments. 


\section{Off Balance Sheet FX Interventions}

We also construct a measure that, in addition to the adjustment for valuation effects and income flows, accounts for off-balance sheet (non-spot) operations, as:

$$
F X I_{j, t} \equiv \Delta N F C A_{j, t}-\left(\Delta^{v a l} N F C A_{j, t}+\Delta^{i n c} N F C A_{j, t}\right)+O B S_{i, t}
$$

where $O B S_{i, t}$ include changes in aggregate short and long positions in forwards and futures in foreign currencies vis-a-vis the domestic currency (including the forward leg of currency swaps), and financial instruments denominated in foreign currency but settled by other means (e.g., in domestic currency), as reported in the International Reserves and Foreign Currency Liquidity Template.

Table 11: Valuation adjustments

\begin{tabular}{|c|c|c|c|c|}
\hline Type of asset & $\begin{array}{l}\text { Currency } \\
\text { Structure }\end{array}$ & $\begin{array}{l}\text { Asset/Maturity } \\
\text { Structure }\end{array}$ & $\begin{array}{l}\text { Valuation } \\
\text { adjustment }\end{array}$ & $\begin{array}{l}\text { Estimated } \\
\text { Income }\end{array}$ \\
\hline \multicolumn{5}{|l|}{ Official Reserve Assets } \\
\hline \multicolumn{5}{|l|}{ Foreign currency reserves } \\
\hline Securities & \multirow{2}{*}{$\begin{array}{l}\text { Currency } \\
\text { shares from } \\
\text { COFER } \\
\text { (US\$, €, £, } \\
¥, \mathrm{~A} \$, \mathrm{C} \$, \\
\text { and SFranc) }{ }^{1}\end{array}$} & $\begin{array}{l}\text { 10-year } \\
\text { sovereign } \\
\text { bonds }^{2}\end{array}$ & $\begin{array}{lc}\text { Implicit } & \text { mar- } \\
\text { ket } & \text { value } \\
\text { based } & \text { on } \\
10-\text { year } & \text { rate }^{3}\end{array}$ & $\begin{array}{l}\text { 10-year } \\
\text { coupon rate }\end{array}$ \\
\hline Total currency and deposits & & 3-month CD & None & $\begin{array}{l}\text { 3-month } \\
\text { interbank } \\
\text { rate }\end{array}$ \\
\hline IMF reserve position & \multirow{2}{*}{ SDR basket } & \multirow{2}{*}{ SDR basket } & \multirow{2}{*}{$\begin{array}{l}\text { SDR valua- } \\
\text { tion changes }\end{array}$} & \multirow{2}{*}{$\mathrm{SDR}$ rate } \\
\hline SDRs & & & & \\
\hline Gold & None & None & $\begin{array}{l}\text { Gold Price } \\
\text { variations }\end{array}$ & None \\
\hline Other reserve assets & $\begin{array}{l}\text { Currency } \\
\text { shares from } \\
\text { COFER } \\
\text { (US\$, €, £, } \\
¥, \quad \mathrm{~A} \$, \mathrm{C} \$, \\
\text { and SFranc) }\end{array}$ & $\begin{array}{l}\text { 10-year } \\
\text { sovereign } \\
\text { bonds }\end{array}$ & $\begin{array}{lc}\text { Implicit } & \text { mar- } \\
\text { ket } & \text { value } \\
\text { based } & \text { on } \\
10-\text { year rate }\end{array}$ & $\begin{array}{l}10 \text {-year } \\
\text { coupon rate }\end{array}$ \\
\hline
\end{tabular}

Source: IMF COFER and Data Template on International Reserves /Foreign Currency Liquidity.

${ }^{1}$ Using annual aggregate statistics for the groups of emerging market economies. Weights are adjusted proportionally to add to 1 .

2 As in Dominguez (2012), assumes 10-year maturity holdings.

${ }^{3}$ Computed from the market interest rate.

\section{Annex 2 Capital controls}

Other recent studies have pointed to the possible complementarity between FXI and restrictions on capital mobility. ${ }^{34}$ While this aspect is somewhat beyond the scope of the paper, we discuss this issue briefly, using our set-up and allowing for an interaction term between FXI and different measures of capital controls. Similarly to previous extensions, the interacted term is instrumented separately, and the set of instrumental variables includes the baseline instruments both alone and interacted with capital controls. Specifically:

\footnotetext{
${ }^{34}$ Bayoumi et al. (2014) find that the impact of FXI on the current account is larger for economies with greater restrictions on capital flow mobility.
} 


$$
\begin{aligned}
\log \left(E R_{i t}\right) & =\alpha+\beta \log \left(E R_{i t-1}\right)+\gamma_{3} F \widehat{X I_{i t}^{G D} P}+\gamma_{4}\left(F X I_{i t}^{G D P \times} K C_{i t-1}\right)+\boldsymbol{\delta}^{\prime} \mathbf{X}_{i t}+\eta_{i}+\epsilon_{i t} \\
F X I_{i t}^{G D P} & =a_{3}+b_{3} \log \left(E R_{i t-1}\right)+\mathbf{c}_{3}^{\prime} \mathbf{Z}_{i t}+\mathbf{e}_{3}^{\prime}\left(\mathbf{Z}_{i t} \times K C_{i t-1}\right)+\mathbf{d}_{3}^{\prime} \mathbf{X}_{i t}+u_{i}+v_{i t} \\
F X I_{i t}^{G D P} \times K C_{i t-1} & =a_{4}+b_{4} \log \left(E R_{i t-1}\right)+\mathbf{c}_{4}^{\prime} \mathbf{Z}_{i t}+\mathbf{e}_{4}^{\prime}\left(\mathbf{Z}_{i t} \times K C_{i t-1}\right)+\mathbf{d}_{4}^{\prime} \mathbf{X}_{i t}+w_{i}+z_{i t}
\end{aligned}
$$

The innovation relative to the baseline is the interaction with capital controls $\left(\mathrm{KC}_{i t}\right)$, for which we use three different indices: Schindler (2009), Quinn and Toyoda (2008) and Chinn and Ito (2006). Each index is introduced in levels, or as threshold dummies for high capital controls (using different threshold values). We explore both absolute (time-invariant) and time-varying thresholds based on the period cross-country distribution of the index. Lagged values of capital controls are used to mitigate endogeneity concerns, and the level of capital controls is also included as a control variable.

Our results (not reported) do not lend support to the hypothesis of complementarity between capital controls and FXI; and in some cases they are counter-intuitive, suggesting that the effect of FXI on the exchange rate may decrease with higher levels of capital controls. These results point to the complexity of the relationship between FXI and restrictions on capital mobility, policy tools that could be used as complements or as substitutes of each other. For instance, restrictions on capital flows in the form of 'quotas' could increase the effectiveness of FXI, by reducing the substitutability between domestic and foreign assets. But imposing capital controls could also render FXI unnecessary, if it suffices to maintain exchange rate stability. In the latter case, we would simultaneously observe high capital controls and very low levels of intervention. Furthermore, the set of instruments used in the baseline specification may not be appropriate when exploring the interaction between capital controls and FXI, as reserve accumulation and exchange rate stabilization motives are likely to change in the presence of significant restrictions on capital mobility. 


\section{Appendix}

Figure A1: Correlation between the change in $\log (\mathrm{RER})$ and FXI/GDP

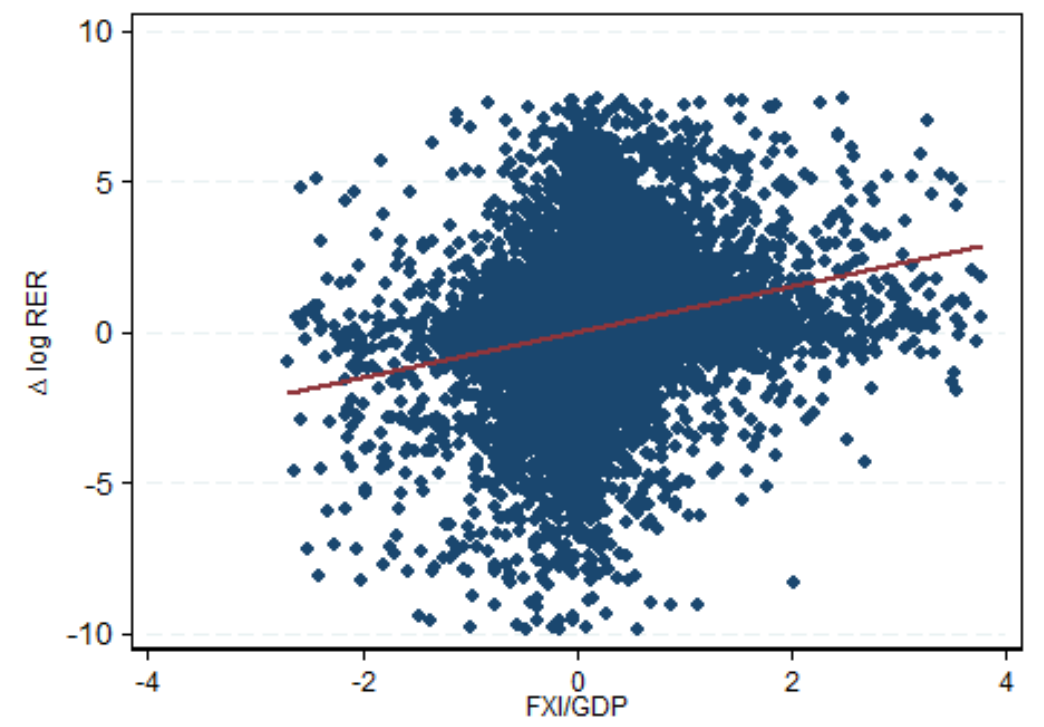

Sources: IMF International Financial Statistics; and authors' calculations

Figure A2: Distribution of FXI/GDP

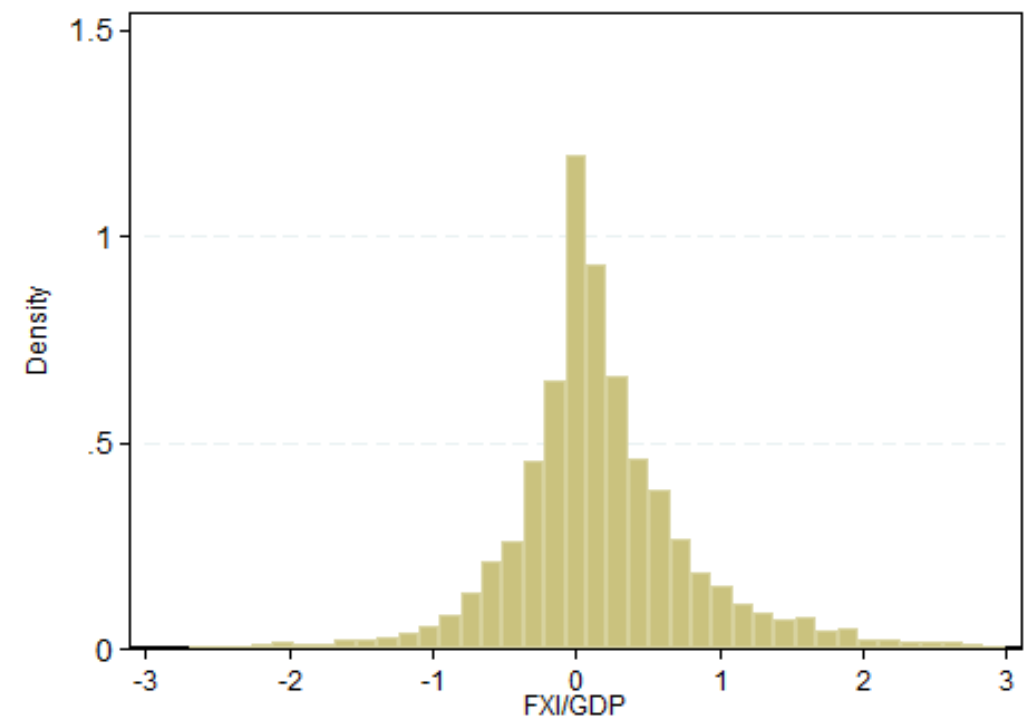

Sources: IMF International Financial Statistics; and authors' calculations 
Table A1: Country list

\begin{tabular}{|c|c|c|c|c|}
\hline & IFS code & Start date & End date & Obs \\
\hline Argentina & 213 & Feb 1996 & Dec 2011 & 173 \\
\hline Armenia & 911 & Feb 1996 & Aug 2013 & 206 \\
\hline Australia & 193 & Feb 1996 & Sep 2013 & 203 \\
\hline Bolivia & 218 & Feb 1996 & Aug 2013 & 208 \\
\hline Brazil & 223 & Feb 1996 & Sep 2013 & 191 \\
\hline Bulgaria & 918 & Aug 1997 & Sep 2013 & 184 \\
\hline Canada & 156 & Feb 1996 & Aug 2013 & 209 \\
\hline Chile & 228 & Feb 1996 & Sep 2013 & 209 \\
\hline China & 924 & Jul 1999 & Sep 2013 & 152 \\
\hline Colombia & 233 & Feb 1996 & Oct 2013 & 210 \\
\hline Costa Rica & 238 & Feb 1996 & Aug 2013 & 211 \\
\hline Croatia & 960 & Feb 1996 & Jun 2013 & 206 \\
\hline Czech Republic & 935 & Feb 1996 & Sep 2013 & 205 \\
\hline Denmark & 128 & Feb 1996 & Sep 2013 & 201 \\
\hline Egypt & 469 & Jan 2001 & Aug 2013 & 149 \\
\hline Guatemala & 258 & Jan 1997 & May 2010 & 160 \\
\hline Honduras & 268 & Jan 2001 & Sep 2013 & 153 \\
\hline Hong Kong & 532 & Jan 1997 & Aug 2013 & 122 \\
\hline Hungary & 944 & Jan 2000 & Sep 2013 & 152 \\
\hline India & 534 & Jan 1999 & Sep 2013 & 177 \\
\hline Indonesia & 536 & Feb 1996 & Dec 2012 & 173 \\
\hline Israel & 436 & Feb 1996 & Jan 2010 & 168 \\
\hline Japan & 158 & Feb 1996 & Aug 2013 & 135 \\
\hline Kazakhstan & 916 & Jul 2000 & Dec 2012 & 142 \\
\hline Kenya & 664 & Mar 2002 & Dec 2011 & 115 \\
\hline Korea & 542 & Feb 1996 & Aug 2013 & 194 \\
\hline Lithuania & 946 & Feb 1996 & Sep 2013 & 206 \\
\hline Malaysia & 548 & Feb 1996 & Jul 2013 & 191 \\
\hline Mexico & 273 & Apr 1996 & Sep 2013 & 207 \\
\hline Moldova & 921 & Mar 2000 & Oct 2013 & 159 \\
\hline New Zealand & 196 & Feb 1996 & Jun 2011 & 177 \\
\hline Nicaragua & 278 & Jan 2006 & Dec 2012 & 84 \\
\hline Nigeria & 694 & Jun 2007 & Dec 2012 & 62 \\
\hline Norway & 142 & Feb 1996 & Jan 2008 & 141 \\
\hline Pakistan & 564 & Feb 1996 & Sep 2013 & 207 \\
\hline Paraguay & 288 & Feb 1996 & Sep 2013 & 205 \\
\hline Peru & 293 & Feb 1996 & Sep 2013 & 212 \\
\hline Philippines & 566 & Feb 1996 & Apr 2013 & 192 \\
\hline Poland & 964 & Feb 1996 & Sep 2013 & 201 \\
\hline Romania & 968 & Apr 2001 & Sep 2013 & 142 \\
\hline Russia & 922 & Dec 1999 & Sep 2013 & 161 \\
\hline Saudi Arabia & 456 & Feb 1996 & Mar 2013 & 178 \\
\hline South Africa & 199 & Jan 1999 & Oct 2013 & 162 \\
\hline Sri Lanka & 524 & Feb 1996 & Jan 2013 & 202 \\
\hline Sweden & 144 & Feb 1996 & Sep 2013 & 205 \\
\hline Switzerland & 146 & Jan 1998 & Sep 2013 & 171 \\
\hline Thailand & 578 & Feb 1996 & Oct 2013 & 200 \\
\hline Turkey & 186 & Nov 1999 & Sep 2013 & 130 \\
\hline Ukraine & 926 & Nov 1998 & Sep 2013 & 166 \\
\hline United Kingdom & 112 & Feb 1996 & Sep 2013 & 199 \\
\hline Uruguay & 298 & Feb 1996 & Sep 2013 & 197 \\
\hline Vietnam & 582 & Feb 1996 & Jul 2013 & 184 \\
\hline
\end{tabular}


Table A2: Variables list and definition

\begin{tabular}{|c|c|c|}
\hline Variable Name & Definition & Source \\
\hline NER & Nominal bilateral exchange rate (increase $=$ appreciation) & IFS \\
\hline RER & $\begin{array}{l}\text { Real bilateral exchange rate, computed from NER and CPI, in- } \\
\text { crease=appreciation }\end{array}$ & CPI: datastream, IFS \\
\hline REER & Real effective exchange rate (increase $=$ appreciation) & IFS \\
\hline FXI/GDP & $\begin{array}{l}\text { Foreign exchange intervention (FXI) proxy, computed as the change in the } \\
\text { central bank's net foreign assets, normalized by annual HP trend US dollar } \\
\text { GDP (in percentage points) }\end{array}$ & IFS \\
\hline $\begin{array}{l}\text { FXI/GDP (net of val- } \\
\text { uation gains and in- } \\
\text { come flows) }\end{array}$ & $\begin{array}{l}\text { FXI proxy, computed as the change in the central bank's net foreign assets } \\
\text { and adjusted for valuation gains and income flows, normalized by annual HP } \\
\text { trend US dollar GDP (in percentage points) }\end{array}$ & $\begin{array}{l}\text { Authors calculations based } \\
\text { on IFS; IMFs Data Tem- } \\
\text { plate on International Re- } \\
\text { serves and Foreign Cur- } \\
\text { rency Liquidity; and IMFs } \\
\text { COFER dataset }\end{array}$ \\
\hline $\begin{array}{l}\text { FXI/GDP (net of val- } \\
\text { uation and income } \\
\text { flows and including } \\
\text { off-balance sheet op- } \\
\text { erations) }\end{array}$ & $\begin{array}{l}\text { FXI proxy, computed as the change in the central bank's net foreign assets, } \\
\text { adjusted for valuation gains and income flows, and including off-balance sheet } \\
\text { operations; normalized by annual HP trend US dollar GDP (in percentage } \\
\text { points) }\end{array}$ & \\
\hline FXI/GDP (gross) & $\begin{array}{l}\text { FXI proxy, computed as the change in the central bank's gross foreign assets, } \\
\text { normalized by annual HP trend US dollar GDP (in percentage points) }\end{array}$ & IFS \\
\hline FXI/M2 & $\begin{array}{l}\text { FXI proxy, computed as the change in the central bank's net foreign assets, } \\
\text { normalized by annual smoothed broad money ( } \% \text { points) }\end{array}$ & IFS \\
\hline FXI/FSS (narrow) & $\begin{array}{l}\text { FXI proxy, computed as the change in the central bank's net foreign assets, } \\
\text { normalized by annual smoothed financial sector size (IFS data) ( } \% \text { points) }\end{array}$ & IFS \\
\hline FXI/FSS (broad) & $\begin{array}{l}\text { FXI proxy, computed as the change in the central bank's net foreign assets, } \\
\text { normalized by annual smoothed financial sector size (WB data) (\% points) }\end{array}$ & IFS, World Bank GFDD \\
\hline $\mathrm{FXI} / \mathrm{MS}$ & $\begin{array}{l}\text { Foreign exchange intervention, computed as the change in the central bank's } \\
\text { net foreign assets, normalized by annual smoothed market size ( } \% \text { points) }\end{array}$ & IFS, World Bank GFDD \\
\hline VIX & Chicago Board Options Exchange Market Volatility Index (VIX) & Haver Analytics \\
\hline $\begin{array}{l}\text { Interest rate differen- } \\
\text { tial }\end{array}$ & $\begin{array}{l}\log (1+\mathrm{i})-\log \left(1+\mathrm{i}^{*}\right) \text {, where } \mathrm{i} \text { is the domestic interest rate and } \mathrm{i}^{*} \text { is the US } \\
\text { interest rate }(\% \text { points })\end{array}$ & IFS \\
\hline Change in M2 & $\begin{array}{l}\text { Change in broad money over the past month, normalized by smoothed GDP } \\
(\% \text { points })\end{array}$ & IFS \\
\hline $\begin{array}{l}\text { Financial dollariza- } \\
\text { tion }\end{array}$ & Share of domestic deposits denominated in foreign currency (\% points) & $\begin{array}{l}\text { Levy-Yeyati (2006), IMF } \\
\text { country desks }\end{array}$ \\
\hline Imports coverage & Central bank's net foreign assets over yearly imports (\% points) & IFS, DOTS \\
\hline Low imports coverage & $\begin{array}{l}\text { Time-varying dummy equal to } 1 \text { if imports coverage are below the cross- } \\
\text { country first quartile }\end{array}$ & IFS, DOTS \\
\hline $\begin{array}{l}\text { Broad money cover- } \\
\text { age }\end{array}$ & Central bank's net foreign assets over M2 (\% points) & IFS \\
\hline $\begin{array}{l}\text { GDP per capita (dif- } \\
\text { ferential) }\end{array}$ & $\begin{array}{l}\text { Difference bewteen domestic GDP per capita in country i and the U.S., in } \\
\text { logarithm. }\end{array}$ & WEO \\
\hline $\begin{array}{l}\text { Expected GDP } \\
\text { growth (differential) }\end{array}$ & Current year expected GDP growth, relative to the U.S. & Consensus Forecast \\
\hline Trade balance & Expc & DOT \\
\hline Trade openness & $\begin{array}{l}\text { Rratio of yearly imports plus exports over GDP, smoothed by a } 3 \text { years moving } \\
\text { average (\% points) }\end{array}$ & DOTS, IFS \\
\hline $\mathrm{EPFR} / \mathrm{GDP}$ & $\begin{array}{l}\text { Sum of yearly flows of funds towards other countries, normalized by GDP of } \\
\text { other countries ( } \% \text { points) }\end{array}$ & EPFR Flow of Funds \\
\hline EMBI & EMBI global spread (basis points) & Bloom \\
\hline Inflation & Yearly inflation rate over past 12 months, computed from CPI ( $\%$ points) & IFS, Datastream \\
\hline Capital controls & Quinn \& Toyoda index for capital controls (the higher, the more open) & Quinn \& Toyoda, IMF \\
\hline Commodity prices & Price indexes for food, metal and energy prices & IFS \\
\hline
\end{tabular}


Table A3: FXI normalizations correlations

\begin{tabular}{lccccc}
\hline \hline & & & & \\
& FXI/GDP & FXI/M2 & FXI/FSS (IFS) & FXI/FSS (WB) & FXI/MS \\
\hline FXI/GDP & 1 & & & & \\
FXI/M2 & $0.819^{* * *}$ & 1 & & & \\
FXI/FSS (IFS) & $0.848^{* * *}$ & $0.843^{* * *}$ & 1 & 1 & \\
FXI/FSS (WB) & $0.794^{* * *}$ & $0.804^{* * *}$ & $0.942^{* * *}$ & & 1 \\
& & & & $0.473^{* * *}$ & 1 \\
FXI/MS & $0.325^{* * *}$ & $0.467^{* * *}$ & $0.413^{* * *}$ & & \\
\hline \hline
\end{tabular}

Table A4: Different normalizations: First stage

\begin{tabular}{|c|c|c|c|c|c|}
\hline & $\begin{array}{c}(1) \\
\text { FXI/GDP }\end{array}$ & $\begin{array}{c}(2) \\
\text { FXI/M2 }\end{array}$ & $\begin{array}{c}(3) \\
\text { FXI/FSS(IFS) }\end{array}$ & $\begin{array}{c}(4) \\
\text { FXI/FSS(WB) }\end{array}$ & $\begin{array}{c}(5) \\
\text { FXI/MS }\end{array}$ \\
\hline Exchange rate (lagged) & $\begin{array}{c}-0.003^{* *} \\
(0.001)\end{array}$ & $\begin{array}{c}-0.013^{* * *} \\
(0.005)\end{array}$ & $\begin{array}{c}-0.005^{* *} \\
(0.002)\end{array}$ & $\begin{array}{c}-0.005 \\
(0.003)\end{array}$ & $\begin{array}{c}-0.037^{* *} \\
(0.018)\end{array}$ \\
\hline VIX & $\begin{array}{c}-0.005^{* *} \\
(0.002)\end{array}$ & $\begin{array}{l}-0.007 \\
(0.005)\end{array}$ & $\begin{array}{c}-0.008^{* *} \\
(0.003)\end{array}$ & $\begin{array}{c}-0.012^{* *} \\
(0.004)\end{array}$ & $\begin{array}{l}-0.041 \\
(0.025)\end{array}$ \\
\hline Interest rate (differential) & $\begin{array}{c}-0.095^{* *} \\
(0.037)\end{array}$ & $\begin{array}{l}-0.256 \\
(0.153)\end{array}$ & $\begin{array}{c}-0.195^{* *} \\
(0.083)\end{array}$ & $\begin{array}{c}-0.237^{* *} \\
(0.109)\end{array}$ & $\begin{array}{l}-0.881 \\
(0.990)\end{array}$ \\
\hline Change in $\mathrm{M} 2 / \mathrm{GDP}$ & $\begin{array}{c}0.104^{* * *} \\
(0.022)\end{array}$ & $\begin{array}{c}0.305^{* * *} \\
(0.073)\end{array}$ & $\begin{array}{c}0.202^{* * *} \\
(0.045)\end{array}$ & $\begin{array}{c}0.318^{* * *} \\
(0.078)\end{array}$ & $\begin{array}{c}1.136^{* * *} \\
(0.379)\end{array}$ \\
\hline Financial dollarization $\times$ VIX & $\begin{array}{c}-0.011^{* *} \\
(0.005)\end{array}$ & $\begin{array}{c}-0.071^{* * *} \\
(0.023)\end{array}$ & $\begin{array}{c}-0.028^{* * *} \\
(0.010)\end{array}$ & $\begin{array}{c}-0.038^{* * *} \\
(0.013)\end{array}$ & $\begin{array}{c}-0.205^{* *} \\
(0.099)\end{array}$ \\
\hline Import coverage (lagged) & $\begin{array}{c}-0.252^{*} \\
(0.142)\end{array}$ & $\begin{array}{l}-0.663 \\
(0.510)\end{array}$ & $\begin{array}{l}-0.268 \\
(0.339)\end{array}$ & $\begin{array}{l}-0.118 \\
(0.419)\end{array}$ & $\begin{array}{c}0.920 \\
(1.345)\end{array}$ \\
\hline Low import coverage (lagged) & $\begin{array}{c}0.035 \\
(0.042)\end{array}$ & $\begin{array}{c}0.022 \\
(0.125)\end{array}$ & $\begin{array}{c}0.022 \\
(0.091)\end{array}$ & $\begin{array}{c}0.088 \\
(0.106)\end{array}$ & $\begin{array}{c}0.722 \\
(0.785)\end{array}$ \\
\hline Broad money coverage (lagged) & $\begin{array}{c}-0.021 \\
(0.145)\end{array}$ & $\begin{array}{l}-0.443 \\
(0.979) \\
\end{array}$ & $\begin{array}{l}-0.242 \\
(0.563)\end{array}$ & $\begin{array}{l}-0.463 \\
(0.756)\end{array}$ & $\begin{array}{l}-4.733 \\
(3.013) \\
\end{array}$ \\
\hline Commodity prices & Yes & Yes & Yes & Yes & Yes \\
\hline Country fixed effects & Yes & Yes & Yes & Yes & Yes \\
\hline Observations & 9149 & 9053 & 8741 & 8424 & 8515 \\
\hline Countries & 52 & 52 & 52 & 48 & 50 \\
\hline$R^{2}$ & 0.10 & 0.11 & 0.14 & 0.11 & 0.15 \\
\hline F stat & 8.18 & 7.46 & 7.64 & 7.11 & 2.87 \\
\hline F p-value & 0.00 & 0.00 & 0.00 & 0.00 & 0.02 \\
\hline
\end{tabular}

Standard errors robust to autocorrelation and heteroskedasticity in parentheses

${ }^{*} p<0.10,{ }^{* *} p<0.05,{ }^{* * *} p<0.01$ 
Table A5: Excluding de jure and de facto pegs: Second stage



Standard errors robust to autocorrelation and heteroskedasticity in parentheses

${ }^{*} p<0.10,{ }^{* *} p<0.05,{ }^{* * *} p<0.01$ 
Table A6: Robustness to capital controls: Second stage

\begin{tabular}{|c|c|c|c|c|c|c|}
\hline & $(2)$ & $(3)$ & (4) & $(5)$ & (6) \\
\hline & \multicolumn{2}{|c|}{$\begin{array}{l}\text { Excluding change in } \\
\text { capital controls }\end{array}$} & \multicolumn{4}{|c|}{ Full sample } \\
\hline FXI/GDP & $\begin{array}{c}-1.231^{* * *} \\
(0.335)\end{array}$ & $\begin{array}{c}-1.392^{* * *} \\
(0.405)\end{array}$ & $\begin{array}{c}-1.686^{* * *} \\
(0.450)\end{array}$ & $\begin{array}{c}-1.650^{* * *} \\
(0.443)\end{array}$ & $\begin{array}{c}-2.004^{* * *} \\
(0.543)\end{array}$ & $\begin{array}{c}-2.007^{* * *} \\
(0.545)\end{array}$ \\
\hline Dependent variable (lagged) & $\begin{array}{c}0.967^{* * *} \\
(0.004)\end{array}$ & $\begin{array}{c}0.954^{* * *} \\
(0.004)\end{array}$ & $\begin{array}{c}0.966^{* * *} \\
(0.004)\end{array}$ & $\begin{array}{c}0.966^{* * *} \\
(0.004)\end{array}$ & $\begin{array}{c}0.951^{* * *} \\
(0.005)\end{array}$ & $\begin{array}{c}0.951^{* * *} \\
(0.005)\end{array}$ \\
\hline VIX & $\begin{array}{c}-0.039^{* * *} \\
(0.006)\end{array}$ & $\begin{array}{c}-0.046^{* * *} \\
(0.007)\end{array}$ & & & & \\
\hline VIX $\times$ Capital controls & & & $\begin{array}{c}-0.048^{* * *} \\
(0.008)\end{array}$ & $\begin{array}{c}-0.039^{* * *} \\
(0.007)\end{array}$ & $\begin{array}{c}-0.064^{* * *} \\
(0.009)\end{array}$ & $\begin{array}{c}-0.065^{* * *} \\
(0.009)\end{array}$ \\
\hline Interest rate (differential) & $\begin{array}{c}0.070 \\
(0.122)\end{array}$ & $\begin{array}{c}0.167 \\
(0.143)\end{array}$ & & & & \\
\hline Interest rate diff. $\times$ Capital controls & & & $\begin{array}{l}-0.030 \\
(0.187)\end{array}$ & $\begin{array}{c}0.016 \\
(0.187)\end{array}$ & $\begin{array}{c}0.057 \\
(0.205)\end{array}$ & $\begin{array}{c}0.057 \\
(0.205)\end{array}$ \\
\hline GDP per capita (differential) & & $\begin{array}{c}3.512^{* * *} \\
(0.969)\end{array}$ & & & $\begin{array}{c}4.557^{* * *} \\
(1.124)\end{array}$ & $\begin{array}{c}4.511^{* * *} \\
(1.128)\end{array}$ \\
\hline Expected GDP growth (differential) & & $\begin{array}{c}0.123^{* * *} \\
(0.028)\end{array}$ & & & $\begin{array}{c}0.111^{* * *} \\
(0.031)\end{array}$ & $\begin{array}{c}0.110^{* * *} \\
(0.031)\end{array}$ \\
\hline Trade Balance (lagged) & & $\begin{array}{c}0.009 \\
(0.095)\end{array}$ & & & $\begin{array}{l}-0.096 \\
(0.118)\end{array}$ & $\begin{array}{l}-0.095 \\
(0.118)\end{array}$ \\
\hline Trade Openness & & $\begin{array}{c}0.265^{* * *} \\
(0.093)\end{array}$ & & & $\begin{array}{c}0.500^{* * *} \\
(0.126)\end{array}$ & $\begin{array}{c}0.504^{* * *} \\
(0.127)\end{array}$ \\
\hline EPFR/GDP & & $\begin{array}{c}1.930^{* * *} \\
(0.331)\end{array}$ & & & $\begin{array}{c}2.032^{* * *} \\
(0.353)\end{array}$ & $\begin{array}{c}3.229^{* * *} \\
(1.078)\end{array}$ \\
\hline EPFR $\times$ Capital controls & & & & $\begin{array}{c}2.564^{* * *} \\
(0.437)\end{array}$ & & $\begin{array}{l}-1.574 \\
(1.368) \\
\end{array}$ \\
\hline Commodity prices & Yes & Yes & Yes & Yes & Yes & Yes \\
\hline Country fixed effects & Yes & Yes & Yes & Yes & Yes & Yes \\
\hline Observations & 8284 & 7474 & 8020 & 8020 & 7467 & 7467 \\
\hline Countries & 52 & 51 & 50 & 50 & 47 & 47 \\
\hline$R^{2}$ & 0.98 & 0.98 & 0.98 & 0.98 & 0.97 & 0.97 \\
\hline $\mathrm{J}$ p-value & 0.20 & 0.22 & 0.22 & 0.45 & 0.70 & 0.69 \\
\hline Stock \& Yogo stat & 17.24 & 13.10 & 12.77 & 12.90 & 10.19 & 10.11 \\
\hline
\end{tabular}

Standard errors robust to autocorrelation and heteroskedasticity in parentheses

${ }^{*} p<0.10,{ }^{* *} p<0.05,{ }^{* * *} p<0.01$ 
Table A7: Robustness to interest rate policy: Second stage

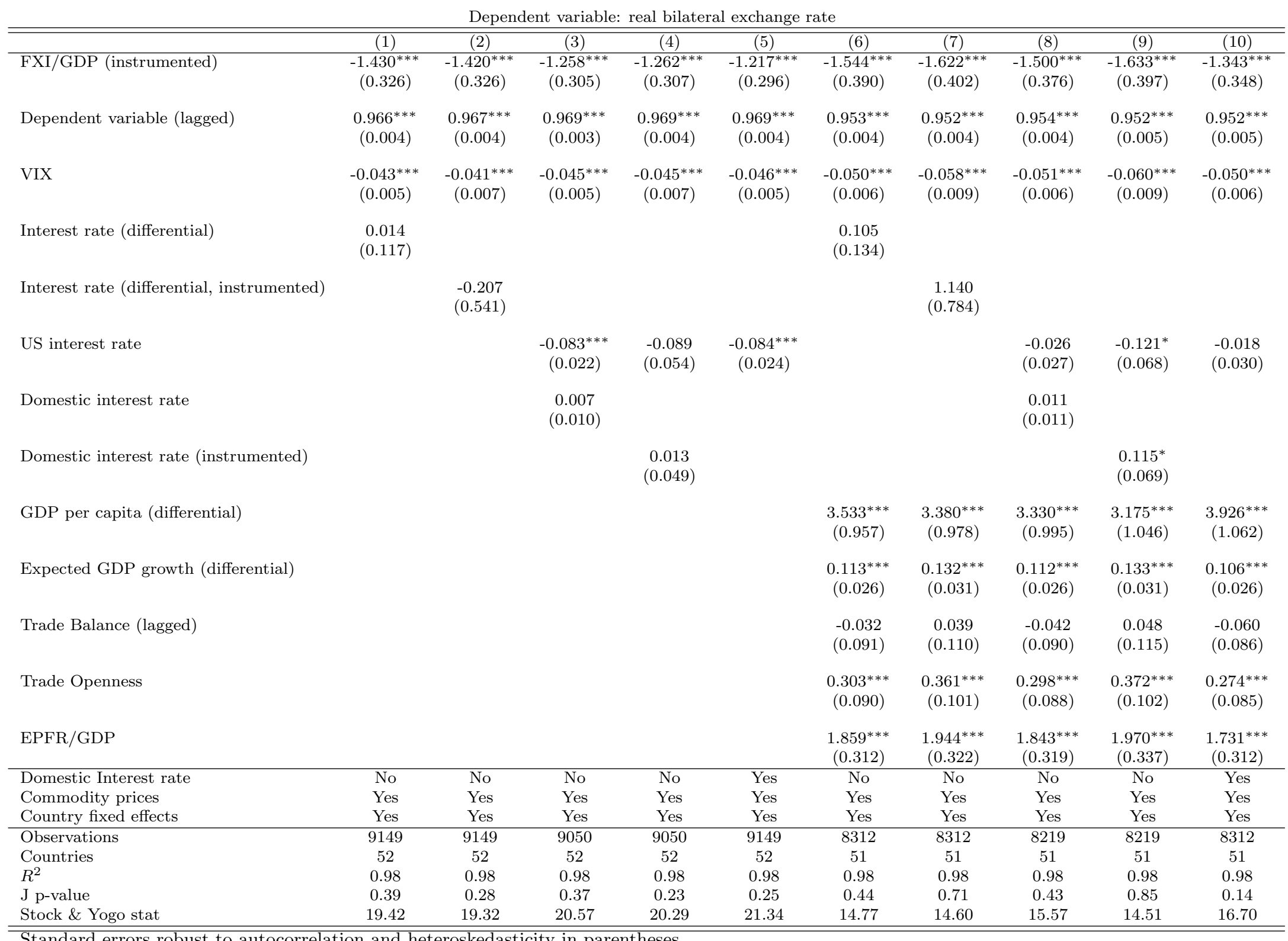

Standard errors robust to autocorrelation and heteroskedasticity in parentheses 
Table A8: FXI measure: Second stage

Dependent variable: real bilateral exchange rate

\begin{tabular}{|c|c|c|c|c|}
\hline & $\begin{array}{c}\text { (1) } \\
\text { Valuation }\end{array}$ & $\begin{array}{c}(2) \\
\text { Valuation \& } \\
\text { off-balance sheet }\end{array}$ & Gross reserves & $\begin{array}{c}(4) \\
\text { Dropping small } \\
\text { FXI } 10 \%\end{array}$ \\
\hline FXI/GDP (instrumented) & $\begin{array}{c}-1.343^{* * *} \\
(0.307)\end{array}$ & $\begin{array}{c}-1.364^{* * *} \\
(0.315)\end{array}$ & $\begin{array}{c}-1.571^{* * *} \\
(0.369)\end{array}$ & $\begin{array}{c}-1.288^{* * *} \\
(0.320)\end{array}$ \\
\hline Dependent variable (lagged) & $\begin{array}{c}0.969^{* * *} \\
(0.003)\end{array}$ & $\begin{array}{c}0.968^{* * *} \\
(0.003)\end{array}$ & $\begin{array}{c}0.968^{* * *} \\
(0.003)\end{array}$ & $\begin{array}{c}0.967^{* * *} \\
(0.004)\end{array}$ \\
\hline VIX & $\begin{array}{c}-0.046^{* * *} \\
(0.006)\end{array}$ & $\begin{array}{c}-0.047^{* * *} \\
(0.006)\end{array}$ & $\begin{array}{c}-0.043^{* * *} \\
(0.006)\end{array}$ & $\begin{array}{c}-0.039^{* * *} \\
(0.006)\end{array}$ \\
\hline Interest rate (differential) & $\begin{array}{c}0.070 \\
(0.110)\end{array}$ & $\begin{array}{c}0.046 \\
(0.114)\end{array}$ & $\begin{array}{c}0.035 \\
(0.113)\end{array}$ & $\begin{array}{c}-0.037 \\
(0.114)\end{array}$ \\
\hline Commodity prices & Yes & Yes & Yes & Yes \\
\hline Country fixed effects & Yes & Yes & Yes & Yes \\
\hline Observations & 9012 & 9011 & 9103 & 8234 \\
\hline Countries & 52 & 52 & 52 & 52 \\
\hline$R^{2}$ & 0.98 & 0.98 & 0.98 & 0.98 \\
\hline $\mathrm{J}$ p-value & 0.25 & 0.28 & 0.18 & 0.24 \\
\hline Stock \& Yogo stat & 20.78 & 19.07 & 19.34 & 18.54 \\
\hline
\end{tabular}

Standard errors robust to autocorrelation and heteroskedasticity in parentheses

${ }^{*} p<0.10,{ }^{* *} p<0.05,{ }^{* * *} p<0.01$

Table A9: FXI measures correlations

\begin{tabular}{lcccc}
\hline \hline & FXI & FXI (val adj) & FXI (val adj + off-BS) & FXI $(\Delta$ Reserves $)$ \\
\hline FXI & 1 & & \\
FXI (val adj) & $0.732^{* * *}$ & 1 & 1 \\
FXI (val adj + off-BS) & $0.722^{* * *}$ & $0.968^{* * *}$ & \\
FXI ( $\Delta$ Reserves) & $0.688^{* * *}$ & $0.493^{* * *}$ & $0.470^{* * *}$ & 1 \\
\hline \hline
\end{tabular}

See Annex 1 for details on the construction of these proxies. 
Table A10: Further robustness checks

Dependent variable: real bilateral exchange rate

\begin{tabular}{|c|c|c|c|c|c|c|}
\hline & \multicolumn{4}{|c|}{ Second stage results } & \multicolumn{2}{|c|}{ Instrumentation tests } \\
\hline & FXI/GDP & Std Dev & Obs & $R^{2}$ & $\mathrm{~J}$ p-value & Stock \& Yogo stat \\
\hline Whole sample & $-1.684^{* * *}$ & 0.521 & 9815 & 0.97 & 0.31 & 7.44 \\
\hline Dropping $1 \%$ outliers & $-1.430^{* * *}$ & 0.326 & 9149 & 0.98 & 0.39 & 19.42 \\
\hline Winsorized data $1 \%$ & $-2.203^{* * *}$ & 0.468 & 9815 & 0.96 & 0.13 & 17.52 \\
\hline Winsorized data $2 \%$ & $-2.397^{* * *}$ & 0.503 & 9815 & 0.96 & 0.12 & 18.94 \\
\hline No financial crisis & $-1.493^{* * *}$ & 0.377 & 8642 & 0.98 & 0.25 & 16.62 \\
\hline No Asian crisis & $-1.381^{* * *}$ & 0.327 & 7588 & 0.98 & 0.42 & 18.37 \\
\hline Dropping largest FXI 5\% & $-1.497^{* * *}$ & 0.379 & 8692 & 0.98 & 0.35 & 15.86 \\
\hline Dropping largest FXI $10 \%$ & $-1.569^{* * *}$ & 0.422 & 8235 & 0.98 & 0.33 & 15.04 \\
\hline Substituting VIX by EPFR & $-1.055^{* * *}$ & 0.302 & 9149 & 0.98 & 0.02 & 18.97 \\
\hline Substituting VIX by EMBI & $-1.246^{* * *}$ & 0.323 & 8421 & 0.98 & 0.23 & 18.73 \\
\hline Adding EMBI & $-1.384^{* * *}$ & 0.329 & 8421 & 0.98 & 0.01 & 15.94 \\
\hline Country specific coeffs for VIX & $-1.318^{* * *}$ & 0.341 & 9149 & 0.98 & 0.08 & 17.55 \\
\hline Country specific coeffs for IR & $-1.462^{* * *}$ & 0.330 & 9149 & 0.98 & 0.36 & 19.09 \\
\hline Country specific coeffs for VIX \& IR & $-1.284^{* * *}$ & 0.331 & 9149 & 0.98 & 0.36 & 17.86 \\
\hline$\Delta \mathrm{RER}$ & $-0.959^{* * *}$ & 0.278 & 9149 & -0.16 & 0.05 & 21.44 \\
\hline Adding REER gap & $-1.543^{* * *}$ & 0.399 & 7833 & 0.98 & 0.46 & 13.65 \\
\hline Sample period 2003-2013 & $-1.241^{* * *}$ & 0.330 & 5977 & 0.97 & 0.15 & 15.69 \\
\hline Only inflation-targeting countries & $-1.744^{* * *}$ & 0.876 & 3772 & 0.97 & 0.00 & 6.46 \\
\hline
\end{tabular}

Standard errors robust to autocorrelation and heteroskedasticity 
Table A11: Dynamic effects: Second stage

\begin{tabular}{|c|c|c|c|c|c|c|c|c|c|c|c|c|}
\hline \multicolumn{13}{|c|}{ Dependent variable: exchange rate } \\
\hline \multirow{3}{*}{$\begin{array}{l}\text { Exchange rate definition: } \\
\text { FXI/GDP }\end{array}$} & (1) & $\begin{array}{c}(2) \\
\text { luding de iu }\end{array}$ & pegs $(3)$ & $(4)$ & $\begin{array}{c}(5) \\
\text { cluding de in }\end{array}$ & e pegs $(6)$ & \multirow{2}{*}{\multicolumn{3}{|c|}{$\begin{array}{c}(7) \quad(8) \\
\text { Including de jure pegs }\end{array}$}} & \multicolumn{3}{|c|}{ 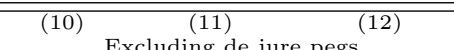 } \\
\hline & Nominal & Real & Real effective & Nominal & Real & Real effective & & & & Nominal & $\begin{array}{l}\text { Real } \\
\text { Realuang }\end{array}$ & Real effective \\
\hline & $\begin{array}{c}-1.694^{* * *} \\
(0.359)\end{array}$ & $\begin{array}{c}-1.431^{* * *} \\
(0.333)\end{array}$ & $\begin{array}{c}-1.113^{* * *} \\
(0.228)\end{array}$ & $\begin{array}{l}-1.913^{* * *} \\
(0.534)\end{array}$ & $\begin{array}{l}-1.757^{* * *} \\
(0.505)\end{array}$ & $\begin{array}{c}-1.446^{* * *} \\
(0.417)\end{array}$ & $\begin{array}{l}-1.868^{* * *} \\
(0.452)\end{array}$ & $\begin{array}{c}-1.511^{* * *} \\
(0.393)\end{array}$ & $\begin{array}{c}-1.221^{* * *} \\
(0.303)\end{array}$ & $\begin{array}{l}-2.028^{* * *} \\
(0.653)\end{array}$ & $\begin{array}{c}-1.699^{* * *} \\
(0.580)\end{array}$ & $\begin{array}{c}-1.316^{* * *} \\
(0.461)\end{array}$ \\
\hline FXI/GDP (lagged) & $\begin{array}{l}0.113^{* *} \\
(0.054)\end{array}$ & $\begin{array}{l}0.097^{*} \\
(0.051)\end{array}$ & $\begin{array}{c}0.132^{* * *} \\
(0.035)\end{array}$ & $\begin{array}{l}0.119^{*} \\
(0.064)\end{array}$ & $\begin{array}{c}0.094 \\
(0.063)\end{array}$ & $\begin{array}{c}0.177^{* * *} \\
(0.047)\end{array}$ & $\begin{array}{l}0.115^{*} \\
(0.061)\end{array}$ & $\begin{array}{l}0.102^{*} \\
(0.055)\end{array}$ & $\begin{array}{c}0.148^{* * *} \\
(0.039)\end{array}$ & $\begin{array}{c}0.105 \\
(0.069)\end{array}$ & $\begin{array}{l}0.090 \\
(0.065)\end{array}$ & $\begin{array}{c}0.170^{* * *} \\
(0.046)\end{array}$ \\
\hline FXI/GDP (2nd lag) & $\begin{array}{c}0.005 \\
(0.054)\end{array}$ & $\begin{array}{l}-0.005 \\
(0.051)\end{array}$ & $\begin{array}{c}0.071^{* *} \\
(0.032)\end{array}$ & $\begin{array}{l}-0.026 \\
(0.064)\end{array}$ & $\begin{array}{l}-0.045 \\
(0.061)\end{array}$ & $\begin{array}{l}0.081^{*} \\
(0.042)\end{array}$ & $\begin{array}{l}-0.012 \\
(0.061)\end{array}$ & $\begin{array}{l}-0.011 \\
(0.055)\end{array}$ & $\begin{array}{c}0.080^{* *} \\
(0.036)\end{array}$ & $\begin{array}{l}-0.049 \\
(0.069)\end{array}$ & $\begin{array}{l}-0.051 \\
(0.064)\end{array}$ & $\begin{array}{c}0.068 \\
(0.043)\end{array}$ \\
\hline FXI/GDP (3rd lag) & $\begin{array}{c}0.137^{* * *} \\
(0.050)\end{array}$ & $\begin{array}{l}0.113^{* *} \\
(0.047)\end{array}$ & $\begin{array}{c}0.025 \\
(0.031)\end{array}$ & $\begin{array}{l}0.154^{* *} \\
(0.063)\end{array}$ & $\begin{array}{l}0.121^{* *} \\
(0.061)\end{array}$ & $\begin{array}{c}0.048 \\
(0.046)\end{array}$ & $\begin{array}{l}0.127^{* *} \\
(0.057)\end{array}$ & $\begin{array}{l}0.108^{* *} \\
(0.052)\end{array}$ & $\begin{array}{c}0.023 \\
(0.037)\end{array}$ & $\begin{array}{l}0.128^{*} \\
(0.069)\end{array}$ & $\begin{array}{l}0.108^{*} \\
(0.063)\end{array}$ & $\begin{array}{c}0.022 \\
(0.047)\end{array}$ \\
\hline Dependent variable (lagged) & $\begin{array}{c}0.976^{* * *} \\
(0.003)\end{array}$ & $\begin{array}{c}0.966^{* * *} \\
(0.004)\end{array}$ & $\begin{array}{c}0.964^{* * *} \\
(0.004)\end{array}$ & $\begin{array}{c}0.971^{* * *} \\
(0.003)\end{array}$ & $\begin{array}{c}0.960^{* * *} \\
(0.004)\end{array}$ & $\begin{array}{c}0.961^{* * *} \\
(0.005)\end{array}$ & $\begin{array}{c}0.974^{* * *} \\
(0.003)\end{array}$ & $\begin{array}{c}0.952^{* * *} \\
(0.004)\end{array}$ & $\begin{array}{c}0.960^{* * *} \\
(0.005)\end{array}$ & $\begin{array}{c}0.968^{* * *} \\
(0.004)\end{array}$ & $\begin{array}{c}0.945^{* * *} \\
(0.005)\end{array}$ & $\begin{array}{c}0.958^{* * *} \\
(0.005)\end{array}$ \\
\hline VIX & $\begin{array}{c}-0.058^{* * *} \\
(0.006)\end{array}$ & $\begin{array}{c}-0.042^{* * *} \\
(0.005)\end{array}$ & $\begin{array}{l}-0.012^{* * *} \\
(0.005)\end{array}$ & $\begin{array}{c}-0.061^{* * *} \\
(0.007)\end{array}$ & $\begin{array}{c}-0.045^{* * *} \\
(0.006)\end{array}$ & $\begin{array}{c}-0.018^{* * *} \\
(0.005)\end{array}$ & $\begin{array}{c}-0.063^{* * *} \\
(0.007)\end{array}$ & $\begin{array}{c}-0.048^{* * *} \\
(0.006)\end{array}$ & $\begin{array}{c}-0.017^{* * *} \\
(0.005)\end{array}$ & $\begin{array}{c}-0.065^{* * *} \\
(0.008)\end{array}$ & $\begin{array}{c}-0.050^{* * *} \\
(0.007)\end{array}$ & $\begin{array}{c}-0.019^{* * *} \\
(0.006)\end{array}$ \\
\hline Interest rate (differential) & $\begin{array}{l}-0.247^{*} \\
(0.128)\end{array}$ & $\begin{array}{l}0.046 \\
(0.113)\end{array}$ & $\begin{array}{l}0.116 \\
(0.114)\end{array}$ & $\begin{array}{c}-0.350^{* *} \\
(0.151)\end{array}$ & $\begin{array}{c}0.007 \\
(0.130)\end{array}$ & $\begin{array}{l}0.090 \\
(0.130)\end{array}$ & $\begin{array}{l}-0.239 \\
(0.147)\end{array}$ & $\begin{array}{c}0.160 \\
(0.127)\end{array}$ & $\begin{array}{l}0.224^{*} \\
(0.131)\end{array}$ & $\begin{array}{c}-0.349^{* *} \\
(0.172)\end{array}$ & $\begin{array}{c}0.130 \\
(0.144)\end{array}$ & $\begin{array}{c}0.191 \\
(0.142)\end{array}$ \\
\hline Inflation rate (differential) & $\begin{array}{c}0.049^{* * *} \\
(0.012)\end{array}$ & & & $\begin{array}{c}0.061^{* * *} \\
(0.013)\end{array}$ & & & $\begin{array}{c}0.054^{* * *} \\
(0.013)\end{array}$ & & & $\begin{array}{c}0.067^{* * *} \\
(0.014)\end{array}$ & & \\
\hline GDP per capita (differential) & & & & & & & $\begin{array}{l}1.460 \\
(0.940)\end{array}$ & $\begin{array}{c}3.998^{* * *} \\
(0.936)\end{array}$ & $\begin{array}{c}0.889 \\
(0.671)\end{array}$ & $\begin{array}{l}1.220 \\
(1.073)\end{array}$ & $\begin{array}{c}4.037^{* * *} \\
(1.044)\end{array}$ & $\begin{array}{c}1.087 \\
(0.735)\end{array}$ \\
\hline Expected GDP growth (differential) & & & & & & & $\begin{array}{c}0.115^{* * *} \\
(0.028)\end{array}$ & $\begin{array}{c}0.099^{* * *} \\
(0.026)\end{array}$ & $\begin{array}{c}0.079^{* * *} \\
(0.020)\end{array}$ & $\begin{array}{c}0.122^{* * *} \\
(0.032)\end{array}$ & $\begin{array}{c}0.105^{* * *} \\
(0.029)\end{array}$ & $\begin{array}{c}0.078^{* * *} \\
(0.022)\end{array}$ \\
\hline Trade Balance (lagged) & & & & & & & $\begin{array}{c}0.121 \\
(0.100)\end{array}$ & $\begin{array}{l}-0.057 \\
(0.090)\end{array}$ & $\begin{array}{l}0.179^{* *} \\
(0.077)\end{array}$ & $\begin{array}{c}0.130 \\
(0.116)\end{array}$ & $\begin{array}{l}-0.054 \\
(0.103)\end{array}$ & $\begin{array}{l}0.168^{*} \\
(0.089)\end{array}$ \\
\hline Trade Openness & & & & & & & $\begin{array}{l}0.234^{* *} \\
(0.099)\end{array}$ & $\begin{array}{c}0.285^{* * *} \\
(0.090)\end{array}$ & $\begin{array}{c}0.037 \\
(0.062)\end{array}$ & $\begin{array}{l}0.304^{* *} \\
(0.120)\end{array}$ & $\begin{array}{c}0.415^{* * *} \\
(0.112)\end{array}$ & $\begin{array}{c}0.078 \\
(0.076)\end{array}$ \\
\hline EPFR/GDP & & & & & & & $\begin{array}{c}1.602^{* * *} \\
(0.317)\end{array}$ & $\begin{array}{c}1.860^{* * *} \\
(0.311)\end{array}$ & $\begin{array}{c}0.927^{* * *} \\
(0.233)\end{array}$ & $\begin{array}{c}1.677^{* * *} \\
(0.342)\end{array}$ & $\begin{array}{c}1.985^{* * *} \\
(0.337)\end{array}$ & $\begin{array}{c}1.035^{* * *} \\
(0.251)\end{array}$ \\
\hline Commodity prices & Yes & Yes & Yes & Yes & Yes & Yes & Yes & Yes & Yes & Yes & Yes & Yes \\
\hline Country fixed effects & Yes & Yes & Yes & Yes & Yes & Yes & Yes & Yes & Yes & Yes & Yes & Yes \\
\hline Observations & 8533 & 8880 & 8668 & 7522 & 7817 & 7380 & 7761 & 8072 & 7646 & 6929 & 7171 & 6797 \\
\hline Countries & 55 & 52 & 55 & 48 & 48 & 48 & 51 & 51 & 51 & 46 & 46 & 46 \\
\hline$R^{2}$ & 0.98 & 0.98 & 0.98 & 0.98 & 0.98 & 0.97 & 0.98 & 0.98 & 0.97 & 0.97 & 0.98 & 0.97 \\
\hline $\mathrm{J}$ p-value & 0.50 & 0.50 & 0.13 & 0.18 & 0.14 & 0.03 & 0.32 & 0.40 & 0.01 & 0.32 & 0.26 & 0.00 \\
\hline Stock \& Yogo stat & 19.17 & 20.01 & 19.71 & 11.57 & 12.27 & 10.38 & 13.74 & 15.38 & 13.68 & 8.80 & 9.66 & 8.14 \\
\hline Half-life of FXI/GDP effect (months) & 23 & 17 & 14 & 20 & 16 & 12 & 22 & 12 & 12 & 20 & 12 & 12 \\
\hline
\end{tabular}

${ }^{*} p<0.10,{ }^{* *} p<0.05,{ }^{* * *} p<0.01$ 
Table A12: Dynamic effects: Second stage, with lagged controls

\begin{tabular}{|c|c|c|c|c|c|c|c|c|c|c|c|c|}
\hline \multicolumn{13}{|c|}{ Dependent variable: exchange rate } \\
\hline & (1) & \multicolumn{2}{|c|}{$\begin{array}{c}(2) \\
\text { Including de jure pegs }\end{array}$} & $(4)$ & \multicolumn{2}{|c|}{$\begin{array}{l}(5) \\
\text { Excluding de jure pegs }\end{array}$} & & \multicolumn{2}{|c|}{$\begin{array}{c}(8) \\
\text { Including de jure pegs }\end{array}$} & \multicolumn{3}{|c|}{$\begin{array}{cc}(10) & (11) \\
\text { Excluding de jure pegs } & (12)\end{array}$} \\
\hline & Nominal & Real & Real effective & Nominal & $\begin{array}{l}\text { cluding de ju } \\
\text { Real }\end{array}$ & $\begin{array}{l}\text { e pegs } \\
\text { Real effective }\end{array}$ & Nominal & $\begin{array}{l}\text { cluding de ju } \\
\text { Real }\end{array}$ & $\begin{array}{l}\text { e pegs } \\
\text { Real effective }\end{array}$ & Nominal & $\begin{array}{l}\text { cluding de j1 } \\
\text { Real }\end{array}$ & $\begin{array}{l}\text { e pegs } \\
\text { Real effective }\end{array}$ \\
\hline FXI/GDP & $\begin{array}{c}-1.615^{* * *} \\
(0.344)\end{array}$ & $\begin{array}{c}-1.407^{* * *} \\
(0.324)\end{array}$ & $\begin{array}{c}-1.114^{* * * *} \\
(0.222)\end{array}$ & $\begin{array}{c}-1.820^{* * *} \\
(0.506)\end{array}$ & $\begin{array}{c}-1.677^{\text {*** }} \\
(0.484)\end{array}$ & $\begin{array}{c}-1.405^{* * *} \\
(0.397)\end{array}$ & $\begin{array}{c}-2.061^{* * *} \\
(0.484)\end{array}$ & $\begin{array}{c}-1.764^{* * *} \\
(0.434)\end{array}$ & $\begin{array}{c}-1.494^{* * *} \\
(0.334)\end{array}$ & $\begin{array}{c}-2.056^{* * *} \\
(0.661)\end{array}$ & $\begin{array}{c}-1.789^{* * *} \\
(0.604)\end{array}$ & $\begin{array}{c}-1.498^{* * *} \\
(0.477)\end{array}$ \\
\hline FXI/GDP (lagged) & $\begin{array}{c}0.128^{* *} \\
(0.052)\end{array}$ & $\begin{array}{c}0.124^{* *} \\
(0.049)\end{array}$ & $\begin{array}{c}0.122^{* * *} \\
(0.035)\end{array}$ & $\begin{array}{c}0.132^{* *} \\
(0.061)\end{array}$ & $\begin{array}{l}0.125^{* * *} \\
(0.059)\end{array}$ & $\begin{array}{c}0.162^{* * *} \\
(0.047)\end{array}$ & $\begin{array}{c}0.141^{* *} \\
(0.062)\end{array}$ & $\begin{array}{c}0.142^{* *} \\
(0.057)\end{array}$ & $\begin{array}{c}0.144^{* * *} \\
(0.042)\end{array}$ & $\begin{array}{l}0.119^{*} \\
(0.066)\end{array}$ & $\begin{array}{c}0.126^{* *} \\
(0.063)\end{array}$ & $\begin{array}{c}0.152^{* * *} \\
(0.049)\end{array}$ \\
\hline FXI/GDP (2nd lag) & $\begin{array}{c}0.022 \\
(0.053)\end{array}$ & $\begin{array}{c}0.018 \\
(0.049)\end{array}$ & $\begin{array}{c}0.076^{* *} \\
(0.032)\end{array}$ & $\begin{array}{l}-0.007 \\
(0.062)\end{array}$ & $\begin{array}{l}-0.021 \\
(0.059)\end{array}$ & $\begin{array}{c}0.092^{* *} \\
(0.043)\end{array}$ & $\begin{array}{c}0.036 \\
(0.064)\end{array}$ & $\begin{array}{c}0.032 \\
(0.058)\end{array}$ & $\begin{array}{c}0.098^{* *} \\
(0.040)\end{array}$ & $\begin{array}{l}-0.014 \\
(0.070)\end{array}$ & $\begin{array}{l}-0.017 \\
(0.065)\end{array}$ & $\begin{array}{l}0.082^{*} \\
(0.047)\end{array}$ \\
\hline FXI/GDP (3rd lag) & $\begin{array}{c}0.139^{* * *} \\
(0.050)\end{array}$ & $\begin{array}{c}0.125^{* * *} \\
(0.047)\end{array}$ & $\begin{array}{c}0.036 \\
(0.032)\end{array}$ & $\begin{array}{l}0.159^{* *} \\
(0.062)\end{array}$ & $\begin{array}{l}0.135^{* *} \\
(0.060)\end{array}$ & $\begin{array}{c}0.064 \\
(0.046)\end{array}$ & $\begin{array}{l}0.126^{* *} \\
(0.060)\end{array}$ & $\begin{array}{l}0.114^{* *} \\
(0.055)\end{array}$ & $\begin{array}{c}0.057 \\
(0.041)\end{array}$ & $\begin{array}{l}0.116^{*} \\
(0.068)\end{array}$ & $\begin{array}{l}0.107^{*} \\
(0.064)\end{array}$ & $\begin{array}{c}0.056 \\
(0.049)\end{array}$ \\
\hline Dependent variable (lagged) & $\begin{array}{c}0.978^{* * * *} \\
(0.003)\end{array}$ & $\begin{array}{c}0.966^{* * *} \\
(0.003)\end{array}$ & $\begin{array}{c}0.963^{* * * *} \\
(0.004)\end{array}$ & $\begin{array}{c}0.973^{* * *} \\
(0.003)\end{array}$ & $\begin{array}{c}0.961^{* * *} \\
(0.004)\end{array}$ & $\begin{array}{c}0.960 * * * \\
(0.005)\end{array}$ & $\begin{array}{c}0.975^{* * *} \\
(0.004)\end{array}$ & $\begin{array}{c}0.954^{* * *} \\
(0.004)\end{array}$ & $\begin{array}{c}0.958^{* * *} \\
(0.005)\end{array}$ & $\begin{array}{c}0.970^{* * * *} \\
(0.004)\end{array}$ & $\begin{array}{c}0.948 * * * \\
(0.005)\end{array}$ & $\begin{array}{c}0.957^{* * *} \\
(0.005)\end{array}$ \\
\hline VIX & $\begin{array}{c}-0.138^{* * *} \\
(0.011)\end{array}$ & $\begin{array}{c}-0.134^{* * *} \\
(0.011)\end{array}$ & $\begin{array}{c}-0.019^{* *} \\
(0.007)\end{array}$ & $\begin{array}{c}-0.145^{* * *} \\
(0.012)\end{array}$ & $\begin{array}{c}-0.145^{* * *} \\
(0.012)\end{array}$ & $\begin{array}{c}-0.028^{* * *} \\
(0.009)\end{array}$ & $\begin{array}{c}-0.140^{* * *} \\
(0.013)\end{array}$ & $\begin{array}{c}-0.134^{* * *} \\
(0.012)\end{array}$ & $\begin{array}{c}-0.029^{* * *} \\
(0.009)\end{array}$ & $\begin{array}{c}-0.143^{* * *} \\
(0.013)\end{array}$ & $\begin{array}{c}-0.140^{* * *} \\
(0.012)\end{array}$ & $\begin{array}{c}-0.029^{* * *} \\
(0.009)\end{array}$ \\
\hline Interest rate (differential) & $\begin{array}{c}-0.659^{* * *} \\
(0.209)\end{array}$ & $\begin{array}{c}-0.477^{* * *} \\
(0.179)\end{array}$ & $\begin{array}{c}-0.404^{*} \\
(0.217)\end{array}$ & $\begin{array}{c}-0.828^{* * *} \\
(0.246)\end{array}$ & $\begin{array}{c}-0.599^{* * *} \\
(0.214)\end{array}$ & $\begin{array}{c}-0.502^{*} \\
(0.266)\end{array}$ & $\begin{array}{c}-0.811^{* * *} \\
(0.259)\end{array}$ & $\begin{array}{c}-0.581^{* *} \\
(0.233)\end{array}$ & $\begin{array}{c}-0.551^{*} \\
(0.306)\end{array}$ & $\begin{array}{c}-0.941^{* * *} \\
(0.297)\end{array}$ & $\begin{array}{c}-0.659^{* *} \\
(0.272)\end{array}$ & $\begin{array}{c}-0.610^{*} \\
(0.351)\end{array}$ \\
\hline Inflation rate (differential) & $\begin{array}{c}-0.154^{* * * *} \\
(0.035)\end{array}$ & & & $\begin{array}{c}-0.166^{* * *} \\
(0.038)\end{array}$ & & & $\begin{array}{c}-0.202^{* * *} \\
(0.043)\end{array}$ & & & $\begin{array}{c}-0.208^{* * *} \\
(0.046)\end{array}$ & & \\
\hline VIX (lagged) & & & & $\begin{array}{c}0.099^{* * *} \\
(0.013)\end{array}$ & $\begin{array}{c}0.113^{* * *} \\
(0.013)\end{array}$ & $\begin{array}{l}-0.010 \\
(0.008)\end{array}$ & & & & $\begin{array}{c}0.083^{* * *} \\
(0.014)\end{array}$ & $\begin{array}{c}0.092^{* * *} \\
(0.014)\end{array}$ & $\begin{array}{c}-0.019^{* *} \\
(0.009)\end{array}$ \\
\hline GDP per capita (differential) & & & & & & & $\begin{array}{c}0.102 \\
(0.973)\end{array}$ & $\begin{array}{c}3.040^{* * *} \\
(0.947)\end{array}$ & $\begin{array}{c}0.655 \\
(0.735)\end{array}$ & $\begin{array}{l}-0.003 \\
(1.083)\end{array}$ & $\begin{array}{c}3.131^{* * *} \\
(1.033)\end{array}$ & $\begin{array}{c}0.924 \\
(0.790)\end{array}$ \\
\hline Expected GDP growth (differential) & & & & & & & $\begin{array}{c}0.367^{* * *} \\
(0.068)\end{array}$ & $\begin{array}{c}0.362^{* * *} \\
(0.067)\end{array}$ & $\begin{array}{c}0.183^{* * *} \\
(0.054)\end{array}$ & $\begin{array}{c}0.376^{* * *} \\
(0.072)\end{array}$ & $\begin{array}{c}0.382^{* * *} \\
(0.071)\end{array}$ & $\begin{array}{c}0.201^{* * *} \\
(0.060)\end{array}$ \\
\hline Trade Balance (lagged) & & & & & & & $\begin{array}{c}0.175 \\
(0.117)\end{array}$ & $\begin{array}{l}-0.002 \\
(0.109)\end{array}$ & $\begin{array}{c}0.251^{* * *} \\
(0.092)\end{array}$ & $\begin{array}{c}0.163 \\
(0.131)\end{array}$ & $\begin{array}{l}-0.028 \\
(0.123)\end{array}$ & $\begin{array}{c}0.215^{* *} \\
(0.104)\end{array}$ \\
\hline Trade Openness & & & & & & & $\begin{array}{l}0.247^{* *} \\
(0.102)\end{array}$ & $\begin{array}{c}0.290^{* * *} \\
(0.094)\end{array}$ & $\begin{array}{c}0.081 \\
(0.068)\end{array}$ & $\begin{array}{l}0.268^{* *} \\
(0.117)\end{array}$ & $\begin{array}{c}0.373^{* * *} \\
(0.112)\end{array}$ & $\begin{array}{c}0.104 \\
(0.079)\end{array}$ \\
\hline EPFR/GDP & & & & & & & $\begin{array}{c}2.092^{* * *} \\
(0.353)\end{array}$ & $\begin{array}{c}2.400^{* * *} \\
(0.346)\end{array}$ & $\begin{array}{c}1.046^{* * *} \\
(0.254)\end{array}$ & $\begin{array}{c}2.139 * * * \\
(0.370)\end{array}$ & $\begin{array}{c}2.450^{* * *} \\
(0.367)\end{array}$ & $\begin{array}{c}1.107^{* * *} \\
(0.268)\end{array}$ \\
\hline Commodity price & Yes & Yes & Yes & Yes & Yes & Yes & Yes & Yes & Yes & Yes & Yes & Yes \\
\hline Lagged controls & Yes & Yes & Yes & Yes & Yes & Yes & Yes & Yes & Yes & Yes & Yes & Yes \\
\hline Country fixed effects & Yes & Yes & Yes & Yes & Yes & Yes & Yes & Yes & Yes & Yes & Yes & Yes \\
\hline Observations & 8324 & 8821 & 8610 & 7365 & 7759 & 7323 & 7546 & 7953 & 7532 & 6768 & 7069 & 6700 \\
\hline Countries & 55 & 52 & 55 & 48 & 48 & 48 & 51 & 51 & 51 & 46 & 46 & 46 \\
\hline $\begin{array}{l}R^{2} \\
\mathrm{~J} \text { p-value }\end{array}$ & 0.98 & 0.98 & 0.98 & 0.98 & 0.98 & 0.97 & 0.98 & 0.98 & 0.97 & 0.97 & 0.98 & 0.97 \\
\hline $\begin{array}{l}\text { J p-value } \\
\text { Stock \& Yogo stat }\end{array}$ & $\begin{array}{c}0.32 \\
18.86\end{array}$ & $\begin{array}{c}0.64 \\
19.91\end{array}$ & $\begin{array}{c}0.19 \\
20.02\end{array}$ & $\begin{array}{c}0.22 \\
11.56\end{array}$ & $\begin{array}{c}0.24 \\
12.47\end{array}$ & $\begin{array}{c}0.03 \\
10.77\end{array}$ & $\begin{array}{c}0.25 \\
12.78\end{array}$ & $\begin{array}{c}0.60 \\
14.11\end{array}$ & $\begin{array}{c}0.14 \\
12.95\end{array}$ & $\begin{array}{l}0.26 \\
8.15\end{array}$ & $\begin{array}{l}0.30 \\
9.04\end{array}$ & $\begin{array}{l}0.02 \\
7.81\end{array}$ \\
\hline Half-life of FXI/GDP effect (months) & 23 & 15 & 13 & 20 & 15 & 12 & 22 & 12 & 12 & 20 & 12 & 12 \\
\hline
\end{tabular}

Standard errors robust to autocorrelation and heteroskedasticity in parentheses

Since GDP per capita and Trade Openness are slow moving variables, coefficients for their additional lags are not well identified and are not included in the regressions. 